\title{
Generalized Space-Time Super-Modulation and Its Application to Grant-Free Medium Access
}

\author{
Farhad Mehran, Member, IEEE, Konstantinos Nikitopoulos, Senior Member, IEEE, and Hamid \\ Jafarkhani, Fellow, IEEE
}

\begin{abstract}
In this work, Generalized Space-Time SuperModulation (GSTSM) is introduced which enables the transmission of an additional flexible-rate and highly-reliable information stream concurrently with the conventionally transmitted symbols, without the need for increasing the corresponding packet length. This is attained by jointly exploiting the spatial and temporal dimensions of multiple-antenna systems, which enables efficient detection for conventional and additional information subchannels even in highly correlated channel conditions or AWGN channels. In the context of machine-type communications, GSTSM enables grant-free medium access without transmitting additional headers to convey each machine's signature information. Hence, it is shown that even at an extreme case where the data packets of two users are always colliding, GSTSM offers throughput gains of up to $33 \%$ compared to the best examined header-based scheme. For the same scenario, it is shown that GSTSM based on joint multi-user detection provides throughput gains of up to $2.5 \times$ compared with the case where users' signals are detected independently. In addition, it yields over $90 \%$ improvement in achievable rates compared with the schemes that require centralized medium-access coordination. For both joint and independent signal detection schemes, it is also shown that adopting an iterative detection/decoding approach allows to further improve the throughput gains.
\end{abstract}

Index Terms-Multiple-input multiple-output (MIMO), Spacetime coding, Grant-free, Machine-type communications (MTCs).

\section{INTRODUCTION}

G RANT-FREE medium access is expected to play an essential role for uplink data transmission of the future massive Machine-Type-Communications (MTCs) [2] and cellfree massive Multiple-Input Multiple-Output (MIMO) systems [3]. In grant-free access methods, users can transmit their information without sending scheduling requests and grant transmissions [4]. Hence, it results in considerably lower signalling overhead and transmission latency [5]. Ideally, for maximizing the achievable throughput and reliability, as well as for minimizing the latency of future grant-free MTCs, the receiver should be able to effectively combine the undecoded packets of the same machine. In a multi-user environment, this requires reliable identification of each machine's ID, which should be very well protected. This can be achieved either

This work has been presented in part in [1].

Farhad Mehran and Konstantinos Nikitopoulos are with 5G Innovation Centre, Institute for Communication Systems, University of Surrey, Guildford, GU2 7XH, UK (e-mail:\{f.mehran,k.nikitopoulos\}@ surrey.ac.uk).

Hamid Jafarkhani is with Center for Pervasive Communications and Computing, University of California, Irvine, CA, 92697, USA (email:hamidj@uci.edu).

(Corresponding author: Konstantinos Nikitopoulos.) by transmitting long orthogonal sequences, or by transmitting long and strongly (channel) encoded headers [6]. After reliably identifying the transmitting machine, packet combining techniques can be used to store the unsuccessfully decoded packets, and combine them with the subsequently received packets [7]. In this regard, rateless codes, applied at the physical (PHY) layer, are promising candidates to effectively combine and decode the accumulated information with the most recently received packets [8]. Still, to recover the ratelessly encoded packets, besides the machine's ID, the receiver needs to know the ordered position of the received packet in the sequence of packets. This requires additional signalling and therefore, results in heavier header transmission overhead.

To the best of the authors' knowledge, so far, the only solution for alleviating this signalling overhead is Space-Time Super Modulation (STSM) [9][10]. STSM enables the transmission of additional information on top of the conventional information by exploiting the redundancy of space-time block codes [11][12], and without increasing the corresponding packet length. This enables the encoding of the packet's Signature Information (SI) on top of the encoded blocks, and therefore, obviates the need for transmitting packet headers or preambles. However, the design of STSM relies on exploiting the temporal redundancy of the $2 \times 2$ Alamouti space-time block code [13] and therefore, it sacrifices half of the transmission rate for attaining diversity gain. In addition, stateof-the-art systems may have several antennae at the machine side (even with one RF chain) and a large number of Access Point (AP) antennae. These requirements pose new challenges with adopting STSM [9][10] for state-of-the-art systems and therefore, motivated us to revisit the design of STSM. The focus of the current work is to generalize the concept of STSM to the systems that can exploit arbitrarily large numbers of transmitter/receiver antennae, and are not relying on spacetime block codes. Hence, the so-called Generalized SpaceTime Super-Modulation (GSTSM) jointly exploits the spatial and temporal dimensions of multiple-antenna systems that enables the transmission of highly-reliable additional information with a flexible-rate, without increasing the corresponding block length.

The proposed GSTSM scheme has similarities with the index-modulation and spatial-modulation schemes, [14] since they both use the time and spatial resources to deliver additional information, and since both techniques benefit from the same well-established code design methodologies. Still, there are also some fundamental differences between these two schemes. In particular, as described in [14], index mod- 
ulation conveys information by utilizing the "indices" of the building blocks of a communication system and, in particular, by altering the on/off status of specific transmission entities (e.g., transmit antennas, subcarriers). In contrast, GSTSM does not utilize the concept of "indices" and does not map any information into on/off patterns. Instead, it conveys additional information by super-modulating conventionally modulated information. This is done in a way that any available spacetime resources can be fully exploited, when and if present. Namely GSTSM, in contrast to index modulation, benefits from such resources but it does not necessitate that they are always present. In addition, it does not require from any of the existing entities to be in an "off" mode, that can result in resources under-utilization. For example, Spatial Modulation, perhaps the most well-known form of Index Modulation, is not applicable to single-antenna systems or to MIMO systems with line-of-sight or highly correlated MIMO channels. In contrast, and as we discuss in this paper in detail, GSTSM is also applicable to such scenarios. Another fundamental difference is that, while index modulation tries to find alternatively ways to convey information (by using indices), GSTSM's target is to convey low rate and highly reliable information on the top of conventionally transmitted information. In this context GSTSM does need to use "indices", but instead it uses conventionally transmitted information as a "carrier" that further modulates with low rate and highly reliable information. As are result, and in contrast to existing index modulation techniques, GSTSM can be used for grant-free medium access. The fact that GSTSM does not utilize indices also simplifies the way that the corresponding transmission patterns are designed. As a result, and as we discuss in detail in the paper, the avoidance of indices enables easily benefiting from state-ofthe-art coding schemes (e.g., polar codes), something that to the best of the Authors knowledge, is proposed for first time.

Moreover, as the target is to minimize the probability of a detection error, similar to the conventional coded-modulation schemes that are designed in Euclidean space (such as TrellisCoded Modulation [16] and Multi-Level Codes [17]), GSTSM aims to increase the Euclidean distance between the available transmission patterns/codewords. However, GSTSM does not necessitate to use any particular channel coding scheme as in Trellis-Coded Modulation and its Space-Time variants [18] that are designed based on Convolutional codes. While both of GSTSM's information subchannels are operating without any coding scheme, they can adopt any type of channel codes. Also, unlike Multi-Level Codes in which information message is segmented into several streams and encoded by individual component encoders (that are co-optimized) and then combined, GSTSM handles concurrent transmission of two information streams that can have flexible rates. Therefore, at the receiver, the information bits of the GSTSM subchannels can be decoded individually, rather than relying on iterative multi-stage decoding as in Multi-Level Codes.

Furthermore, we employ GSTSM to reduce the signalling overhead for applications with massive connectivity as in MTC use cases in $6 \mathrm{G}$. This is realized by employing additional low-rate information stream of GSTSM to convey packet's signature information and as a result, enabling headerless transmission of data packets. To the best of authors' knowledge, it is for the first time that for the systems with massive connectivity, the signalling overhead is reduced by introducing transmission scheme that obviates the need for communicating packet headers. In [19], authors address the reduction of signalling overhead for OFDM-based relay systems via OFDM-based Index Modulation approach. In contrast, we are proposing GSTSM concept and for the first time, exploiting its application for reduction of signalling overhead in future MTCs.

We will show that the additional highly-reliable flexible-rate information stream of GSTSM yields significant throughput gains with negligible performance loss compared with traditional uncoded systems that are operating without GSTSM. Also, it will be shown that unlike conventional space-time MIMO coding schemes [20] that are particularly designed for MIMO systems, the additional information subchannel of GSTSM can be reliably recovered even in highly correlated channel conditions or AWGN channels. We will conduct various simulation based studies to validate the performance of GSTSM under different performance affecting parameters and operating scenarios, and present that the highly reliable detection for both of the additional and conventional information streams is enabled by exploiting the receive diversity [21]. It will be shown that by leveraging state-of-the-art coding schemes, GSTSM provides significant throughput gains compared with STSM [9][10] which sacrifices half of the transmission rate for attaining transmit diversity. Moreover, for the first time, such a transmission scheme is utilized in the context of MTCs for enabling uncoordinated, grantfree, and headerless transmission of ratelessly encoded data packets. It will be shown that even in extreme case that data packets of two users are always colliding, the GSTSM-enabled headerless scheme has superior performance compared with the best header-based approach. For collision scenarios, it will be shown that by jointly detecting signals of multiple users, significant throughput gains can be attained compared with the approach where the information message of each user is detected independently (by taking the interfering user's signal as noise). Moreover, it is shown that additional throughput gains can be achieved by utilizing iterative detection/decoding techniques to cancel the effect of inter-user-interference from the received signal. In this work, we focus on uncoordinated transmission scenarios as they are deemed to be the desirable medium-access techniques for MTCs [22], and we will show that the GSTSM-enabled scheme also obviates the need for centralized medium-access coordination. However, GSTSM can be still utilized in coordinated access schemes that are operating based on non-/orthogonal signal transmissions [2].

The contributions of this paper are summarized as follows.

1) We propose the concept of GSTSM that allows to transmit additional flexible-rate information bit stream on top of the conventionally modulated symbols, without the need for increasing the block length. Unlike STSM [9][10], the design of GSTSM is not relying on exploiting temporal redundancy of space-time block codes, and can adopt arbitrarily large number of antennae at the transmitter/receiver sides. 
2) We propose two pattern design rules for GSTSM, and elaborate on the related computational complexities and performance evaluations. We show that by introducing the designed bit-to-pattern mapping to the conventionally modulated symbols, GSTSM not only requires significantly lower number of transmit antenna compared to the conventional spatial modulation for yielding the same throughput increase, but it also works in singleinput single-output systems with AWGN.

3) We derive mathematical framework for GSTSM encoding/decoding for uplink data transmissions in multi-user scenarios, and formulate the related soft-informationbased detection problem for state-of-the-art systems that can adopt soft-information-based packet combining techniques. We then conduct the related performance validations under key parameters.

4) We adopt GSTSM for MTCs to enable headerless and grant-free medium access, and provide a comparative study versus the conventional header-based methods in challenging scenarios. We further benchmark the results against transmission schemes that require centralized coordination, and explore the throughput gains (and the related computational complexity and processing latency tradeoffs) based on joint and independent multi-user detection methods, with and without iterative detector/decoder. We also show that GSTSM can adopt dense constellations for the applications that require high data rates.

The rest of the paper is organized as follows. The concept of the GSTSM, and the required processing at the transmitter/receiver sides are presented in Section II. In Section III, we elaborate on the design rules for the construction of super-modulation patterns. Section IV presents the system model aspects of GSTSM in the context of grant-free multiple access to enable headerless transmissions. In Section V, the performance of GSTSM is evaluated under the various design parameters, as well as in different transmission scenarios, and Section VI concludes the paper.

\section{Generalized Space-Time Super-Modulation} (GSTSM)

The encoding and decoding procedures of GSTSM are presented in this section. As it will be shown, GSTSM can be adopted by systems with various number of transmitting antennae, and arbitrarily large number of receiving antennae to exploit receive diversity. Also, it will be shown that GSTSM can adapt to various density of constellations.

\section{A. GSTSM Encoding}

GSTSM encodes the additional information on top of the conventional information by altering the transmission patterns. This is achieved by elaborating on the ideas of spatial modulation [15] and receive diversity of large MIMO systems [21], as well as by introducing temporal correlation [23] to the transmitted symbol sequences. Similar to spatial modulation, GSTSM exploits the different propagation characteristics of the different transmitting antennae. However, rather than

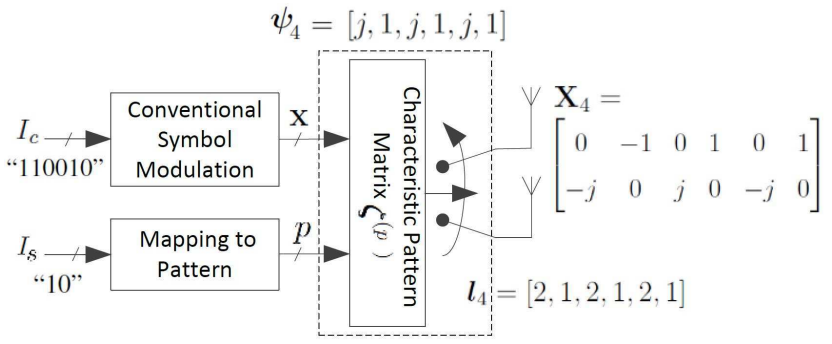

Fig. 1: GSTSM encoder with characteristic pattern matrix $\zeta^{(p)}$, employing two transmitting antennae with one RF chain. The numerical representations are regarding the given example.

allocating distinct bit sequences to each transmitting antenna, GSTSM employs two-dimensional transmission patterns. The additional information is conveyed by mapping the additional information sequence onto these patterns that define how the signal transmission takes place. In particular, one part of the pattern is related to the spatial dimension of the system, and dictates which of the transmitting antennae will be used (i.e., activated) for signal transmission. The other part, is related to the temporal characteristics of the signal, and dictates how the conventionally transmitted information will be phase-rotated. As we show in Section V, the existence of multiple-antennae at the receiver side enables extremely reliable detection of the corresponding patterns (and therefore of the super-modulated information).

In a GSTSM system with $N_{t}$ transmit antennae and a single RF chain, only one transmit antenna is activated for data transmission at any time instant. Based on the additional information bits, a pattern is chosen which determines the phase rotations and indices of the transmitting antennae for the transmission of conventional information symbols. Fig. 1 depicts the block diagram of the encoder. The transmitted information for each GSTSM information block is divided into two subsets. The first subset, $I_{c}$, consists of the conventionally modulated information bits, which are bits that are mapped onto a conventional constellation and would have been transmitted even in the absence of GSTSM. Considering the transmission of conventionally modulated bits of a block length of $K$ symbols, $K \log _{2}|\mathcal{M}|$ bits can be mapped onto conventional complex information symbols $x_{k}$, drawn from a complex constellation $\mathcal{M}$ of size $|\mathcal{M}|$ where $k=1, \ldots, K$. The conventionally modulated symbols can be drawn from any constellation symbol set, e.g., QAM or PSK constellations. Hence, the conventionally modulated word is given by

$$
\mathbf{x}=\left[\begin{array}{lll}
x_{1} & \ldots & x_{K}
\end{array}\right] .
$$

The second subset $I_{s}$ with a block length of $n_{s}$ bits, contains the super-modulated bits (i.e., the additional information) that are going to be encoded by means of GSTSM. The information bits of this stream are mapped onto a pattern $p$ via an appropriate bit-to-pattern mapping. Each pattern $p$ is related to its corresponding characteristic pattern matrix $\boldsymbol{\zeta}^{(p)}$ which describes how the conventionally modulated symbols will be super-modulated. To generate the super-modulated words, it is assumed that each conventional symbol can be further modulated by using one of the $O_{s m}$ prespecified Super- 
Modulation (SM) states, that consist of prespecified phase rotations and active antenna indices. Hence, for a specific pattern $p$, the $N_{t} \times K$ characteristic pattern matrix is defined as $\boldsymbol{\zeta}^{(p)}:=\left(\boldsymbol{\Lambda}^{(p)}, \boldsymbol{\xi}^{(p)}\right)$, where $\boldsymbol{\Lambda}^{(p)}$ and $\boldsymbol{\xi}^{(p)}$ are characteristic antennae activation and phase rotation vectors, respectively. Denoting the phase rotation that is generated according to the characteristic phase rotation vector $\xi^{(p)}$ at time instant $k$ by $\psi_{p, k}$, the phase rotated conventionally modulated symbol is given by $\grave{x}_{p, k}=x_{k} \psi_{p, k}$. The phase rotations for all possible patterns are designed such that the rotated symbols do not coincide for any conventionally transmitted constellation point in order to increase the Euclidean distance between the possible codewords. This makes the patterns distinguishable at the receiver, and boosts the detectability of the additional information in the case of correlated channel conditions. We will elaborate on the design of phase rotation patterns in Section III. The phase rotated symbols are transmitted from the antenna that is specified by the characteristic antenna activation vector $\Lambda^{(p)}$. For the given $\Lambda^{(p)}$, let us denote the index of the active transmitter antenna at time instant $k$ by $l_{p, k} \in\left\{1, \ldots, N_{t}\right\}$. Using the $N_{t}$-dimensional standard basis vector $\mathbf{e}_{l_{p}}$ (i.e., $\mathbf{e}_{1}=[1,0, \ldots, 0]^{T}$ ), the transmitted symbol vector at time instant $k$ is given by $\mathbf{e}_{p, k} \grave{x}_{p, k}$ and can be expressed as

$$
\mathbf{x}_{p, k}=\left[\begin{array}{c}
\mathbf{0}_{\left(l_{p, k}-1\right) \times 1} \\
\grave{x}_{p, k} \\
\mathbf{0}_{\left(N_{t}-l_{p, k}\right) \times 1}
\end{array}\right],
$$

where $\mathbf{x}_{p, k} \in \mathbb{C}^{N_{t} \times 1}$, and $\mathbf{0}_{q \times v}$ is a $q \times v$ all-zero matrix. Therefore, the transmitted codeword is expressed as

$$
\mathbf{X}_{p}=\left[\begin{array}{ccc}
\mathbf{0}_{\left(l_{p, 1}-1\right) \times 1} & \ldots & \mathbf{0}_{\left(l_{p, K}-1\right) \times 1} \\
\grave{x}_{p, 1} & \ldots & \grave{x}_{p, K} \\
\mathbf{0}_{\left(N_{t}-l_{p, 1}\right) \times 1} & \ldots & \mathbf{0}_{\left(N_{t}-l_{p, K}\right) \times 1}
\end{array}\right] .
$$

For GSTSM with $\left|O_{s m}\right|$ super-modulation states and information block length of $K, C=\left|O_{s m}\right|^{K}$ patterns are available. Therefore, without the need to increase the block length, up to $\left\lfloor\log _{2}(C)\right\rfloor$-bit $I_{S}$ can be transmitted per block with an appropriate mapping. Hence, the introduced patterns can result in spectral efficiency increase by up to $\left\lfloor\log _{2}(C)\right\rfloor / K$ bits per channel use. However, there is a tradeoff between the throughput and detection reliability of the additional information stream. In this work, we are conveying additional information stream with low spectral efficiency (to preserve the reliability) that as will be shown, does not have negative impact on reliability of conventional information stream. Let us provide a GSTSM encoding example to present the details, and demonstrate how the transmission patterns are mapped on both temporal and spatial dimensions of a MIMO system. In this example, we assume $K=6, n_{s}=2,\left|O_{s m}\right|=2$, BPSK conventionally modulated symbols, and $N_{t}=2$ with a single RF chain as in Fig. 1 . The $I_{s}$ and $I_{c}$ bits are "10" and "110010", respectively. First, the $I_{c}$ stream is mapped onto BPSK symbols as $\mathbf{x}=\left[\begin{array}{llllll}-1 & -1 & 1 & 1 & -1 & 1\end{array}\right]$. Each possible $I_{s}$ stream has a mapping to a $\zeta^{(p)}$, which dictates how the conventional modulated symbol should be rotated, while also determines the index of active transmitting antenna for transmission of this constellation. We will elaborate on the design of patterns in Section III but for now, the following mapping can be considered as an example:

$$
\begin{aligned}
& \text { "00" } \rightarrow p=1 \rightarrow \boldsymbol{\zeta}^{(1)}=\left(\boldsymbol{\Lambda}^{(1)}, \boldsymbol{\xi}^{(1)}\right)=\left[\begin{array}{llllll}
1 & 1 & 1 & 1 & 1 & 1 \\
0 & 0 & 0 & 0 & 0 & 0
\end{array}\right], \\
& \text { "01" } \rightarrow p=2 \rightarrow \boldsymbol{\zeta}^{(2)}=\left(\boldsymbol{\Lambda}^{(2)}, \boldsymbol{\xi}^{(2)}\right)=\left[\begin{array}{llllll}
1 & 0 & 1 & 0 & 1 & 0 \\
0 & 2 & 0 & 2 & 0 & 2
\end{array}\right], \\
& \text { "11" } \rightarrow p=3 \rightarrow \boldsymbol{\zeta}^{(3)}=\left(\boldsymbol{\Lambda}^{(3)}, \boldsymbol{\xi}^{(3)}\right)=\left[\begin{array}{llllll}
0 & 0 & 0 & 0 & 0 & 0 \\
2 & 2 & 2 & 2 & 2 & 2
\end{array}\right], \\
& \text { "10" } \rightarrow p=4 \rightarrow \boldsymbol{\zeta}^{(4)}=\left(\boldsymbol{\Lambda}^{(4)}, \boldsymbol{\xi}^{(4)}\right)=\left[\begin{array}{llllll}
0 & 1 & 0 & 1 & 0 & 1 \\
2 & 0 & 2 & 0 & 2 & 0
\end{array}\right] .
\end{aligned}
$$

The $I_{s}$ is " 10 ", and therefore $\zeta^{(4)}$ is chosen to determine the mapping on both space and time dimensions. Hence, the activated transmit antenna indices from time instant one to six are $\boldsymbol{l}_{4}=[2,1,2,1,2,1]$, and the elements of phase rotation

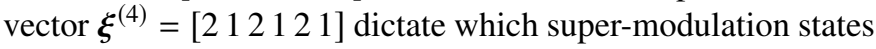
should be used for phase rotations $\psi_{4, k}, \forall k=1, \cdots, K$; therefore, the phase rotations for time instant one to six are given by $\psi_{4,1}=j, \psi_{4,2}=1, \psi_{4,3}=j, \psi_{4,4}=1, \psi_{4,5}=j$, and $\psi_{4,6}=1$. Thus, the transmitted GSTSM word is given by

$$
\mathbf{X}_{4}=\left[\begin{array}{cccccc}
0 & -1 & 0 & 1 & 0 & 1 \\
-j & 0 & j & 0 & -j & 0
\end{array}\right]
$$

\section{B. GSTSM Receiver Processing for Multi-User Scenarios}

Without loss of generality, we consider the extreme case of uncoordinated transmission of $U$ machines that are always colliding in a MIMO uplink system, similar to the $U$-user multiple access channel. Assuming that each user employs $N_{t}$ transmitting antennae, and the AP employs $N_{r}$ receiving antennae, the received signal is given by

$$
\mathbf{Y}=\sum_{u=1}^{U} \mathbf{H}_{u} \mathbf{X}_{p_{u}}+\mathbf{N}
$$

where $\mathbf{H}_{u}$ is the $N_{r} \times N_{t}$ MIMO channel matrix for $u^{t h}$ user, $\mathbf{X}_{p_{u}}$ is the transmitted codeword of user $u$ employing transmission pattern $p_{u}$, and $\mathbf{N}$ is the $N_{r} \times K$ noise matrix consisting of independent and identically distributed (i.i.d.), zero-mean, complex Gaussian samples with variance $2 \sigma_{n}^{2}$. We formulate the Maximum-Likelihood (ML) multi-user detection problem that jointly estimates the transmitted codeword of all $U$ users as

$$
\left[\hat{\mathbf{X}}_{p_{1}}, \cdots, \hat{\mathbf{X}}_{p_{U}}\right]=\arg \min _{\left(\mathbf{x}_{p_{1}}, \cdots, \mathbf{X}_{p_{U}}\right)}\left\{F\left(\mathbf{X}_{p_{1}}, \cdots, \mathbf{X}_{p_{U}}\right)\right\}
$$

where

$$
F\left(\mathbf{X}_{p_{1}}, \cdots, \mathbf{X}_{p_{U}}\right)=\left\|\mathbf{Y}-\sum_{u=1}^{U} \mathbf{H}_{u} \mathbf{X}_{p_{u}}\right\|^{2} .
$$

The minimization in (6) involves exhaustive calculations over all possible codewords for all users which is prohibitively complex to be performed at the receiver side. Hence, using 
algebraic manipulations, the Euclidean cost function in (6) can be calculated as

$$
F\left(\mathbf{X}_{p_{1}}, \cdots, \mathbf{X}_{p_{U}}\right)=\sum_{k=1}^{K}\left\|\mathbf{Y}_{k}-\sum_{u=1}^{U} \mathbf{H}_{u, k} \mathbf{x}_{p_{u}, k}\right\|^{2},
$$

where $\mathbf{x}_{p_{u}, k}$ denotes the possible transmitted symbol vector of User $u$ at time instant $k$, as expressed in (2). Since the terms in summation of (7) are independent, the minimization of $F\left(\mathbf{X}_{p_{1}}, \cdots, \mathbf{X}_{p_{U}}\right)$ can be formulated via the minimization of each term. Thus, denoting the specific patterns assigned for Users 1 to $U$ by $\left(p_{1}, \ldots, p_{U}\right)$, the conventionally modulated symbols for Users 1 to $U$ that minimize (7) for a given $\left(p_{1}, \ldots, p_{U}\right)$ can be formulated as

$$
\begin{gathered}
\left(\hat{x}_{1, k}, \ldots, \hat{x}_{U, k}\right)=\arg \min _{\left(x_{1, k}, \cdots, x_{U, k}\right)}\left\|\mathbf{Y}_{k}-\sum_{u=1}^{U} \mathbf{h}_{p_{u}, k} x_{u, k} \psi_{p_{u}, k}\right\|^{2}, \\
\forall k=1, \ldots, K,
\end{gathered}
$$

where $\mathbf{h}_{p_{u}, k}$ denotes the $N_{r} \times 1$ channel coefficient vector used for transmission of the $u^{\text {th }}$ user's phase-rotated symbol at time instant $k$. Consequently, for specific patterns $\left(p_{1}, \cdots, p_{U}\right)$, the corresponding Euclidean cost function can be expressed as

$$
\begin{gathered}
F_{\text {min }}\left(p_{1}, \cdots, p_{U}\right)=\min \left\{\sum_{k=1}^{K}\left\|\mathbf{Y}_{k}-\sum_{u=1}^{U} \mathbf{h}_{p_{u}, k} x_{u, k} \psi_{p_{u}, k}\right\|^{2}\right\} \\
=\sum_{k=1}^{K}\left\|\mathbf{Y}_{k}-\sum_{u=1}^{U} \mathbf{h}_{p_{u}, k} \hat{x}_{u, k} \psi_{p_{u}, k}\right\|^{2}
\end{gathered}
$$

To jointly estimate the transmitted pattern of the $U$ users, the Euclidean cost (9) should be calculated for each possible pattern combination of all users. Hence, denoting the set of available patterns by $\mathcal{P}$, the ML solution is given by

$$
\left(\hat{p}_{1}, \cdots, \hat{p}_{U}\right)=\arg \min _{\left(p_{1}, \cdots, p_{U}\right) \in \mathcal{P}^{U}}\left\{F_{\min }\left(p_{1}, \cdots, p_{U}\right)\right\} .
$$

Hence, from the solution of (10), the estimate of the conventionally modulated symbols is recovered from (8). The detection problem as formulated here not only allows to "jointly" detect the signals of multiple-users, but it also enables "independent" signal detection for each user by taking into account the inter-user-interference of the rest of the $U-1$ users as noise. Whether we intend to jointly or independently detect the users' signals, the detection problem as formulated above is non-iterative, which can be implemented in an iterative manner as well. In implementation of iterative detection/decoding, when a user decodes its information message successfully, the AP re-runs the detection process by taking into account the recovered symbols of decoded user as an input so as to cancel the effect of inter-user-interference in subsequent round of signal detection. In Section V-B, we elaborate on comparative performance evaluation of iterative and non-iterative schemes for both joint and independent detection approaches.

\section{Packet Combining Methods}

To maximize the throughput, the receiver should be able to store the unsuccessfully decoded packets and efficiently combine them with the most recently received packets and subsequently, re-attempt the decoding. There are several different signal combining techniques available in the literature [7]. To effectively combine the received signals, applying rateless codes on the PHY layer has shown a great deal of attention [8]. In this work, Raptor codes [24] have been used for the rateless coding of the data payload since they are among the most widely used rateless schemes due to their linear time encoding/decoding. Still, the design of GSTSM does not rely on a particular class of combining technique, e.g., IR-HARQ [8] can also be utilized for packet combining in GSTSMenabled systems. The encoding and decoding of Raptor codes are described in [24]. For the decoding, the soft-informationbased belief-propagation decoder is employed. Hence, the softinformation of the conventionally modulated bits need to be calculated and inserted into the decoder. Denoting the parameters of the desirable and interfering users by superscripts $a$ and $b$, respectively, the soft-information of the conventionally modulated bits under the max-log approximation [25] can be calculated as

$$
L_{\left(x_{a, k, q}\right)} \approx
$$

$\min _{\substack{x_{a} \in \mathcal{M}_{q}^{(1)} \\ x_{b} \in \mathcal{M}_{q}^{(0,1)}}}\left\{\left\|\mathbf{Y}_{k}-\mathbf{h}_{\hat{p}_{a}, k} x_{a} \psi_{\hat{p}_{a}, k}-\sum_{\substack{b \in\{1, \ldots, U\} ; \\ b \neq a}} \mathbf{h}_{\hat{p}_{b}, k} x_{b} \psi_{\hat{p}_{b}, k}\right\|^{2} \|^{2}-\right.$ $\min _{\substack{x_{a} \in \mathcal{M}_{q}^{(0)} \\ x_{b} \in \mathcal{M}_{q}^{(0,1)}}}\left\{\left\|\mathbf{Y}_{k}-\mathbf{h}_{\hat{p}_{a}, k} x_{a} \psi_{\hat{p}_{a}, k}-\sum_{\substack{b \in\{1, \ldots, U\} ; \\ b \neq a}} \mathbf{h}_{\hat{p}_{b}, k} x_{b} \psi_{\hat{p}_{b}, k}\right\|^{2} \|^{2}\right\}$,

where $L_{\left(x_{a, k, q}\right)}$ denotes the Log-Likelihood Ratio (LLR) of the $q^{\text {th }}$ bit of User $a$ 's conventional symbol $x$ at time instant $k$, and $\mathcal{M}_{q}^{(0)}$ and $\mathcal{M}_{q}^{(1)}$ are the subsets of the conventional symbols that have the $q^{\text {th }}$ bit equal to 0 and 1 , respectively. The channel coefficients $\mathbf{h}_{\hat{p}_{a}, k}$ and $\mathbf{h}_{\hat{p}_{b}, k}$, as well as the related phase rotations $\psi_{\hat{p}_{a}, k}$ and $\psi_{\hat{p}_{b}, k}$ are chosen according to the solution obtained from the pattern structure of the code. In Section III, we present two pattern designs and explain how they affect packet combining and decoding.

\section{PATTERN DESIGN}

In GSTSM, to transmit the additional information stream $I_{s}$, the pattern $p$ is chosen from the set of available patterns $\mathcal{P}$ which is also referred to as "pattern pool" or codebook. Each pattern $p$ is constructed by selecting one of the available SM states $O_{s m}$ at each time instant, which dictates the phase rotation and the antenna index that is going to be used for transmission of the conventionally modulated symbol. Hence, construction of patterns (i.e., characteristic pattern matrices) depends on block length $K, n_{s}$, and $\left|O_{s m}\right|$ values at each time instant. Therefore, finding patterns that yield 
maximal minimum Euclidean distance between the codewords from the possible $\left(\begin{array}{c}\left|O_{s m}\right|^{K} \\ 2^{n}\end{array}\right)$ pattern codebooks is a non-linear optimization problem which is not feasible to be solved in practice. In this section, two pattern design rules with feasible construction complexity are introduced. As will be shown, these methods yield promising performance although they are not necessarily optimal solutions. In the first approach, the pattern construction rule of [10] is generalized for exploiting both temporal and spatial dimensions of MIMO systems with arbitrarily large numbers of transmitter/receiver antennae. Since this approach allows patterns to be reconstructable provided that the additional and conventional information block lengths are known, we refer to this pattern construction scheme as "Fixed Patterns". The second approach, termed "Polar-Coding-Assisted (PCA)" pattern construction method is designed based upon the potential gains of Polar codes in the short block-length regime [26]. In this approach, Polar-coded sequence of additional information stream determines the phase rotation and antenna index to be used for transmission of conventionally modulated symbols.

\section{A. Fixed Patterns}

Ideally, the pattern design for codebook $\mathcal{P}$ targets maximization of the Euclidean distance between the codewords over all available patterns. To reduce the word error probability, the minimum Euclidean distance $d_{\text {min }}^{2}$ over all possible codewords should be maximized, which is given by

$$
\begin{gathered}
d_{m i n}^{2}\left(\mathbf{X}_{p_{n}}, \mathbf{X}_{p_{m}}\right)=\min \left\{\left\|\mathbf{X}_{p_{n}}-\mathbf{X}_{p_{m}}\right\|^{2}\right\}, \\
\forall \mathbf{X}_{p_{n}}, \mathbf{X}_{p_{m}} \in \mathcal{V} \text {, and } n \neq m,
\end{gathered}
$$

where $\mathcal{V}$ is the set of possible transmitted GSTSM words. Using algebraic manipulations, the Euclidean cost function in (12) can be calculated as

$$
\begin{gathered}
d_{\text {min }}^{2}\left(\mathbf{X}_{p_{n}}, \mathbf{X}_{p_{m}}\right)=\min \left\{\sum_{k=1}^{K}\left\|\mathbf{x}_{p_{n}, k}-\mathbf{x}_{p_{m}, k}\right\|^{2}\right\}, \\
\forall \mathbf{x}_{p_{n}, k}, \mathbf{x}_{p_{m}, k} \in \mathbb{C}^{N_{t} \times 1}, n \neq m, \text { and } \forall k=1, \cdots, K .
\end{gathered}
$$

Since we assume transmitters are operating based on a single RF chain and the channel coefficients does not contribute in Euclidean distance calculation, (13) can be expressed as

$$
\begin{gathered}
d_{m i n}^{2}\left(\mathbf{X}_{p_{n}}, \mathbf{X}_{p_{m}}\right)=\min \left\{\sum_{k=1}^{K}\left\|x_{n, k} \psi_{p_{n}, k}-x_{m, k} \psi_{p_{m}, k}\right\|^{2}\right\}, \\
\forall \psi_{p_{n}, k}, \psi_{p_{m}, k} \in \Phi, \forall x_{n, k}, x_{m, k} \in \mathcal{M}, n \neq m, \text { and } \forall k=1, \cdots, K,
\end{gathered}
$$

where $\Phi$ denotes the set of available phase rotations. The minimization in (14) can be formulated via the minimization of each term since the terms in summation are independent. To increase the symbol's identifiability, fixed phase rotations are used in this approach to maximize the Euclidean distance between the symbols, which can be found by solving

$$
\begin{gathered}
{\left[\hat{\psi}_{p_{n}, k}, \hat{\psi}_{p_{m}, k}\right]=\min _{\psi_{p_{n}, k}, \psi_{p_{m}, k} \in \Phi}\left\{\left\|x_{n, k} \psi_{p_{n}, k}-x_{m, k} \psi_{p_{m}, k}\right\|\right\},} \\
\forall x_{n, k}, x_{m, k} \in \mathcal{M},
\end{gathered}
$$

To improve the identification of $I_{s}, \log _{2}\left|O_{s m}\right|$ bits are mapped on each element of the characteristic pattern matrix $\zeta^{(p)}$ and each of the $n_{s}$ bits redundantly appear $\frac{K \cdot \log _{2}\left|O_{s m}\right|}{n_{s}}$ times in each transmission pattern. For the symmetric PSK with $\left|O_{\text {sm }}\right|$ super-modulation states, the phase rotations that maximize the minimum Euclidean distance between the possible symbols can be calculated analytically from $\psi_{p, k}=$ $\exp \left\{j \phi_{p, k}\right\}=\exp \left\{j \frac{2 \pi}{\left|O_{s m}\right||\mathcal{M}|}\left(\boldsymbol{\xi}_{k}^{(p)}-1\right)\right\}$. For example, for BPSK with $\left|O_{s m}\right|=2$, the phase rotations $\phi \in\left\{0, \frac{\pi}{2}\right\}$ (Fig. 2(a)) result in maximal minimum Euclidean distance of $d_{\text {min }} \approx 1.4$ between the available super-modulated symbols, where $d_{\text {min }}$ corresponds to the Euclidean cost in (15) for given $\hat{\psi}_{p_{n}, k}$ and $\hat{\psi}_{p_{m}, k}$.

For the case of QAM, where it is tedious to find a generic analytical expression, the phase rotations that maximize $d_{\text {min }}$ between two symbols, can be found easily by means of computational methods. For example, for 16-QAM and 64QAM with $\left|O_{s m}\right|=2$, the conducted simulations with step size of $\frac{\pi}{180}$ show that $\phi \in\left\{0, \frac{\pi}{7}\right\}$ and $\left\{0, \frac{\pi}{14}\right\}$ yield maximum $d_{\text {min }} \approx 0.19$ and 0.049 , respectively, e.g., Fig. 2(b) depicts the rotated symbols for 16-QAM. The numerical example provided in Section II-A illustrates an instance of GSTSM encoding based on Fixed Patterns. At the receiver side, the solution of the ML problem formulated in (10) provides the estimate of the transmitted pattern. Hence, in GSTSM-enabled rateless systems, the solution of (10) also determines $\mathbf{h}_{\hat{p}_{a}, k}$ and $\mathbf{h}_{\hat{p}_{b}, k}$, as well as $\psi_{\hat{p}_{a}, k}$ and $\psi_{\hat{p}_{b}, k}$ for the LLR calculations of (11).

When transmission is over fading channels, each pattern is not only mapped onto temporal dimension of conventional symbols, but it also exploits the spatial dimension of multipleantenna systems to improve the pattern identifiability. Let $\mathbf{h}_{p_{n}, k}=\left[h_{p_{n}, k, 1}, \cdots, h_{p_{n}, k, N_{r}}\right]^{T}$, and $h_{p_{n}, k, i}$ denotes the channel coefficient from the active transmitting antenna (assigned for $p_{n}$ ) to the AP's receiving antenna $i$ at time instant $k$. Each of the channel coefficients $h_{p_{n}, k, i}$ can be expressed in terms of its magnitude $\left|h_{p_{n}, k, i}\right|$ and phase $\angle h_{p_{n}, k, i}$ components. Hence, assuming that channel state information is not known at the transmitter side, the channel phase components $\angle h_{p_{n}, k, i}$ are not known. Therefore, with adapting fixed phase rotations it is not possible to maximize the minimum Euclidean distance between $\mathbf{h}_{p_{n}, k} x_{n, k} \psi_{p_{n}, k}$ and $\mathbf{h}_{p_{m}, k} x_{m, k} \psi_{p_{m}, k}$ to minimize the probability of symbol error detection. However, as will be shown in Section V, SM states are still identifiable at the receiver if distinct antenna indices are assigned to them.

\section{B. Polar-Coding-Assisted (PCA) Pattern Construction}

Unlike the Fixed Patterns that rely on a pre-designed set of bit-to-pattern mappings, in PCA-based GSTSM, the mapping used for the construction of characteristic pattern matrix is based on the codeword attained after Polar-coding $I_{s}$. Hence, each bit of the resulting codeword which is denoted by $\bar{I}_{S}$ dictates how the conventionally modulated symbols will be phase-rotated and which of the transmitting antennae will be activated for signal transmission. For the attained $\bar{I}_{s}$, the final transmitted codeword is denoted by $\mathbf{X}_{\left(\bar{I}_{S}\right)}$. Let us provide an example to present the details. In this example, we 


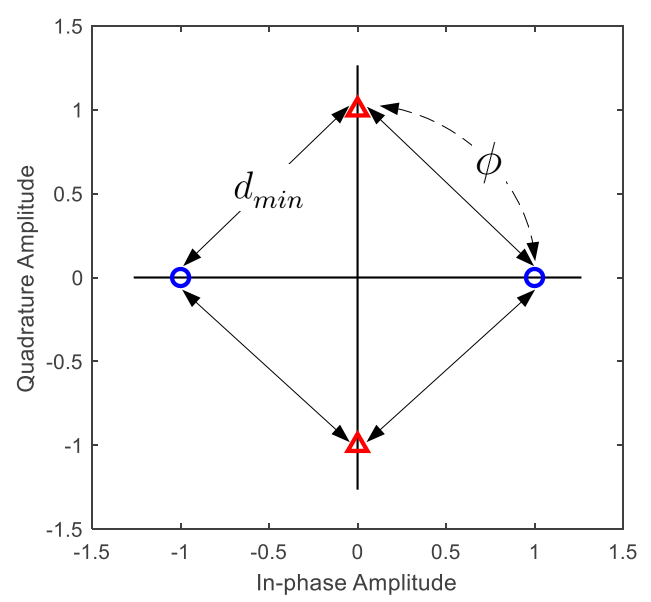

(a)

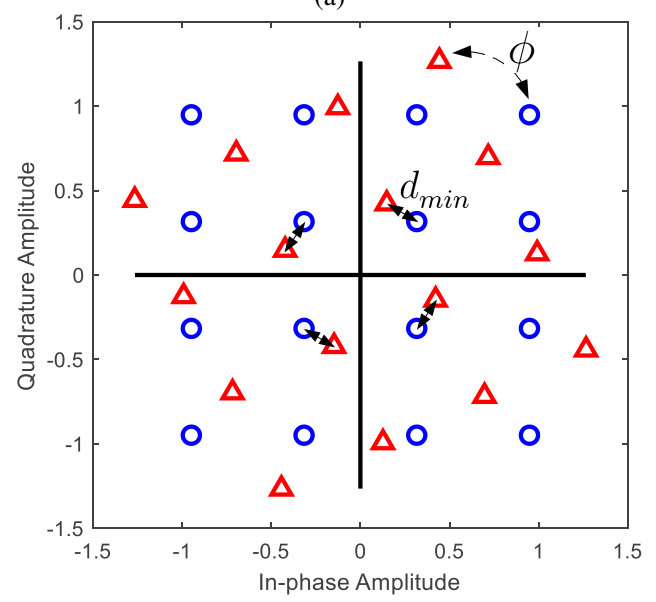

(b)

Fig. 2: Phase rotations for GSTSM schemes with $\left|O_{s m}\right|=2$ for: (a) BPSK; (b) 16-QAM. The constellation points denoted by blue circles and red triangles denote the symbols employing the first and second phase rotations, respectively.

assume a PCA-based GSTSM scheme with $K=8, n_{s}=2$, $\left|O_{s m}\right|=2$, BPSK conventionally modulated symbols, and $N_{t}=2$ with a single RF chain. Let $I_{s}$ and $I_{c}$ be " 10 " and "00111000", respectively. First, $I_{S}$ is Polar-coded, yielding $\bar{I}_{s}=[11110000]$, and $I_{c}$ is mapped onto BPSK symbols, giving $\mathbf{x}=\left[\begin{array}{llllllll}1 & 1 & -1 & -1 & -1 & 1 & 1 & 1\end{array}\right]$. Let us assume that bits 0 and 1 of $\bar{I}_{s}$ indicate that the first and the second phase rotation/antenna index should be used for conveying the conventional symbols. Hence, the phase rotations and the activated transmit antenna indices from time instant one to eight are given by $\psi_{\left(\bar{I}_{s}\right)}=$ $[j, j, j, j, 1,1,1,1]$ and $\boldsymbol{l}_{\left(\bar{I}_{s}\right)}=[2,2,2,2,1,1,1,1]$, respectively. Therefore, the transmitted word is given by

$$
\mathbf{X}_{\left(\bar{I}_{s}\right)}=\left[\begin{array}{cccccccc}
0 & 0 & 0 & 0 & -1 & 1 & 1 & 1 \\
j & j & -j & -j & 0 & 0 & 0 & 0
\end{array}\right] .
$$

For recovering the transmitted patterns, the ML problem (10) as formulated in Section II-B holds only for the Fixed Patterns. To estimate the transmitted pattern of each of the $U$ users for PCA-based schemes, the LLR of the received symbols need to be calculated and fed into Polar decoder for soft-information processing. The soft-information of the Polarcoded sequence under the max-log approximation [25] can be calculated as

$$
\begin{gathered}
L_{\left(\bar{I}_{s, k}^{\alpha}\right)}^{\alpha} \approx \\
\min _{\substack{x_{\alpha}, x_{b} \in \mathcal{M} \\
\mathbf{h}_{k}^{\alpha}=\mathbf{h}_{k}^{\alpha}(1), \psi^{\alpha}=\Phi(1) \\
\mathbf{h}_{k}^{b} \in\left\{\mathbf{h}_{k}^{b}(1), \mathbf{h}_{k}^{b}(2)\right\}, \psi^{b} \in\{\Phi(1), \Phi(2)\}}}\left\{\left\|\mathbf{Y}_{k}-\mathbf{h}_{k}^{\alpha} x_{\alpha} \psi^{\alpha}-\sum_{\substack{b \in\{1, \ldots, U\} ; \\
b \neq \alpha}} \mathbf{h}_{k}^{b} x_{b} \psi^{b}\right\|^{2} \|^{2}-\right.
\end{gathered}
$$$$
\min _{\substack{x_{\alpha}, x_{b} \in \mathcal{M} \\ \mathbf{h}_{k}^{\alpha}=\mathbf{h}_{k}^{\alpha}(2), \psi^{\alpha}=\Phi(2) \\ \mathbf{h}_{k}^{b} \in\left\{\mathbf{h}_{k}^{b}(1), \mathbf{h}_{k}^{b}(2)\right\}, \psi^{b} \in\{\Phi(1), \Phi(2)\}}}\left\{\left\|\mathbf{Y}_{k}-\mathbf{h}_{k}^{\alpha} x_{\alpha} \psi^{\alpha}-\sum_{\substack{b \in\{1, \ldots, U\} ; \\ b \neq \alpha}} \mathbf{h}_{k}^{b} x_{b} \psi^{b}\right\|^{2}\right\},
$$

where $L_{\left(\bar{I}_{s, k}\right)}$ denotes the LLR of the Polar-coded sequence $\bar{I}_{s}$ at time instant $k$, and the superscripts $\alpha$ and $b$ denote the parameters of the desirable and interfering users, respectively. Here, $\mathbf{h}_{k}^{\alpha}(q)$ denotes the $N_{r}$-dimensional channel coefficient vector from User $\alpha$ 's active transmitter antenna $q$ to $\mathrm{AP}$ at time instant $k$, and $\Phi(q)$ denotes the phase rotation assigned for state $q$. The calculated LLRs are inserted into the softinformation-based Polar decoder to estimate the transmitted $I_{s}$. The recovered $I_{s}$ allows to reconstruct the transmitted pattern and accordingly, the estimation of conventionally modulated symbols can be restored from (8). For the GSTSM-enabled rateless systems, the recovered $I_{s}$ also determines the channel coefficients $\mathbf{h}_{\hat{p}_{a}, k}$ and $\mathbf{h}_{\hat{p}_{b}, k}$, as well as the related phase rotations $\psi_{\hat{p}_{a}, k}$ and $\psi_{\hat{p}_{b}, k}$ for the LLR calculations of (11).

In this work, we have chosen Polar codes as they have shown to yield promising performance for short block-length regime [26]. Hence, as Polar codes are binary codes, the resultant super-modulation states are limited to two states. For designing patterns with super-modulation states of higher than two, non-binary channel codes can be used in similar approach as in PCA scheme.

\section{Complexity Analysis}

In this section, we elaborate on the computational complexity of the ML detection for the Fixed and PCA pattern construction methods. For the Fixed Patterns, for calculation of (9), each $\mathbf{h}_{p_{u}, k} \hat{x}_{u, k} \psi_{p_{u}, k}$ term can take up to $\left|O_{s m}\right||\mathcal{M}|$ values, each of which requiring $\left(1+N_{r}\right)$ complex-valued multiplications provided that $\hat{x}_{u, k}$ is first multiplied with $\psi_{p_{u}, k}$. Solving (10) involves $\left(\left|O_{s m} \| \mathcal{M}\right|\right)^{U}$ norm calculations at each time instant, each of which entailing $N_{r}$ complex-valued multiplications. Hence, the maximum complexity of the ML detection for Fixed Patterns is $\left[\left(\left|O_{s m} \| \mathcal{M}\right|\right)^{U}\left(N_{r}+U\left(N_{r}+1\right)\right)\right] K$ per information block. Still, each possible $\mathbf{h}_{p_{u}, k} \hat{x}_{u, k} \psi_{p_{u}, k}$ term can be calculated and stored to be used for calculating the Euclidean cost function of each possible pattern combination in (9). As a result, the incurred complexity can be reduced to $\left[\left(\left|O_{s m}\right||\mathcal{M}|\right)^{U} N_{r}+\left|O_{s m}\right||\mathcal{M}| U\left(N_{r}+1\right)\right] K$ per information block. To keep the overall complexity reasonable, we assume $\left|O_{s m}\right|=2$ for the rest of the paper. For the PCA patterns, assuming $\left|O_{s m}\right|=2$, each $\min \{\cdot\}$ operation of (16) takes 
$|\mathcal{M}|(2|\mathcal{M}|)^{U-1}$ norm operations, each of which requiring $N_{r}$ complex-valued multiplication operations. Hence, since the maximum complexity of calculating each possible $\mathbf{h}_{k}^{\alpha} x_{\alpha} \psi^{\alpha}$ and $\mathbf{h}_{k}^{b} x_{b} \psi^{b}$ term is $\left(1+N_{r}\right)$, the overall complexity for calculating (16) is $\left[(2|\mathcal{M}|)^{U}\left(N_{r}+U\left(N_{r}+1\right)\right)\right] K$ per information block. This can be reduced to $\left[(2|\mathcal{M}|)^{U} N_{r}+2|\mathcal{M}| U\left(N_{r}+1\right)\right] K$ by calculating all possible $\mathbf{h}_{k}^{\alpha} x_{\alpha} \psi^{\alpha}$ and $\mathbf{h}_{k}^{b} x_{b} \psi^{b}$ terms and store them for calculating the Euclidean cost in each of the $\min \{\cdot\}$ arguments.

Still, for both Fixed Patterns and PCA-based schemes, this complexity can be further reduced by solving (10) and (16) for each user independently and taking all the other $(U-1)$ users' signals as noise. In this case, for the Fixed Patterns, solving (10) involves $\left|O_{s m}\right||\mathcal{M}|$ norm calculations at each time instant. Therefore, based on the above mentioned assumptions for imposed complexity for calculation of each norm as well as each $\mathbf{h}_{p_{u}, k} \hat{x}_{u, k} \psi_{p_{u}, k}$ term, the maximum per-user complexity of the ML detection for Fixed Patterns is $\left[\left(\left|O_{s m}\right||\mathcal{M}|\right)\left(2 N_{r}+1\right)\right] K$ per information block, which is $U\left[\left(\left|O_{s m}\right||\mathcal{M}|\right)\left(2 N_{r}+1\right)\right] K$ in total for multi-user cases. For instance, for a GSTSM with $\left|O_{s m}\right|=2,|\mathcal{M}|=2, U=2$, and $N_{r}=1$, the overall complexity of independent multiuser detection is about $33 \%$ of joint multi-user detection approach. For the PCA-based approach, we make similar assumptions as in multi-user joint detection case described above; however, as the signals from the other $(U-1)$ users are taken into account as noise, each $\min \{\cdot\}$ operation of (16) only takes $|\mathcal{M}|$ norm operations. Thus, the overall complexity for calculating $(16)$ is $\left[(2|\mathcal{M}|)\left(2 N_{r}+1\right)\right] K$ per block for each user and $U\left[(2|\mathcal{M}|)\left(2 N_{r}+1\right)\right] K$ for multi-user scenarios. The computational complexity saving of independent detection comes at the cost of performance degradation, but the resulting loss will be negligible when the receiver diversity of large MIMO systems is exploited (as will be shown in Section V).

To overcome the computational complexities incurred by exhaustive search of the ML detection, sphere decoding [27] can be used which transforms the detection problem into a tree search. Also, a great deal of research effort has been put on reducing the computational complexity of sphere-decodingbased detection methods to form them as practical solutions for real systems [27]. In particular, the massively parallelized sphere decoder architectures such as MultiSphere [28] have shown to enable practical and low-latency detection techniques to support uplink multi-user MIMO systems, without compromising their ML performance. While adapting such state-ofthe-art detection schemes for GSTSM is a very interesting topic, it is beyond the scope of this work, and exploring the related performance/complexity tradeoffs for future MTCs with a massive number of machines is the matter of future works.

\section{GSTSM For HeAderless GRant-Free Medium ACCESS}

In this section, we discuss how GSTSM enables headerless and uncoordinated one-shot transmission for MTCs, and without the need for communicating any scheduling request. The scenario considered here assumes that multiple machines communicate with the AP in the uplink as expressed by (4) in Section II-B, and that the AP employs one or more receive antennae. The data payload of each machine is ratelessly encoded, modulated, and transmitted in sets of $K$ symbol blocks. Each machine continuously transmits coded information blocks and the received information is detected, combined, and decoded at the AP. This process continues until the machine receives acknowledgement (ACK) from the AP that confirms that the data has been successfully decoded. Using a dedicated control channel, these ACK messages are broadcasted from AP and it is assumed that they are perfectly received. While the proposed grant-free multiple access scheme is based on rateless codes, the application of GSTSM is transparent to the choice of the channel coding method and it can be used in conjunction with other schemes that detect and combine the received information [7]. Also, the proposed approach is applicable to other wireless networks such as ad-hoc MTCs, as well as to scenarios with random data transmission. To combine and decode the received information blocks at the AP, the receiver needs to know the identity of the transmitting machine, as well as the relative position of the received packet in the whole rateless-coded packet sequence. Hence, additional information bits need to be sent to carry this information, which is referred to as Signature Information (SI). The SI allows each machine to distinguish its signal in received packets that are likely to be contaminated with interuser interference in grant-free communications. Here, the SI consists of two parts. The first segment, consisting of $k_{i}$ bits, is used for providing the identity of the machine. The second part, consisting of $k_{o}$ bits, provides the order of the packet in the rateless-coded packet sequence. To transmit each or all segments of the SI, several methods can be employed. The first approach is based on the mapping of SI bits to orthogonal sequences [29], such as the Zadoff-Chu (ZC) that are used in Random Access Channel (RACH) of LTE [30]. Then, the SI is transmitted by means of preambles/headers that are sent along with the (coded) packets. We have adapted ZC preambles for evaluations and comparisons in Section V.

Also, instead of mapping the SI onto orthogonal sequences, it can be encoded by means of strong and low-rate channel codes [6], modulated similarly to the data packets, and subsequently transmitted as packet preambles/headers. Since the performance of Polar codes is promising for small packet transmissions [26], in this work, we adopted them for the purpose of comparisons with GSTSM-enabled systems. Also, the scheme of Convolutionally-coding packet headers is another preamble-based scheme we examined. Whether the SI is transmitted via mapping to $\mathrm{ZC}$ sequences or via using Polar/Convolutional codes, both methods require the transmission of a packet header that can considerably limit the achievable rate of the system. Instead, by super-modulating the SI on top of the information packets, we obviate the need for transmission of packet headers which as will be shown in Section V, results in significant throughput gains. Also, embedding SI as a hidden clue for identifying user's identity allows to simply detect the user activity by running aforementioned receiver processing in case that energy detector alarms the reception of a signal. 


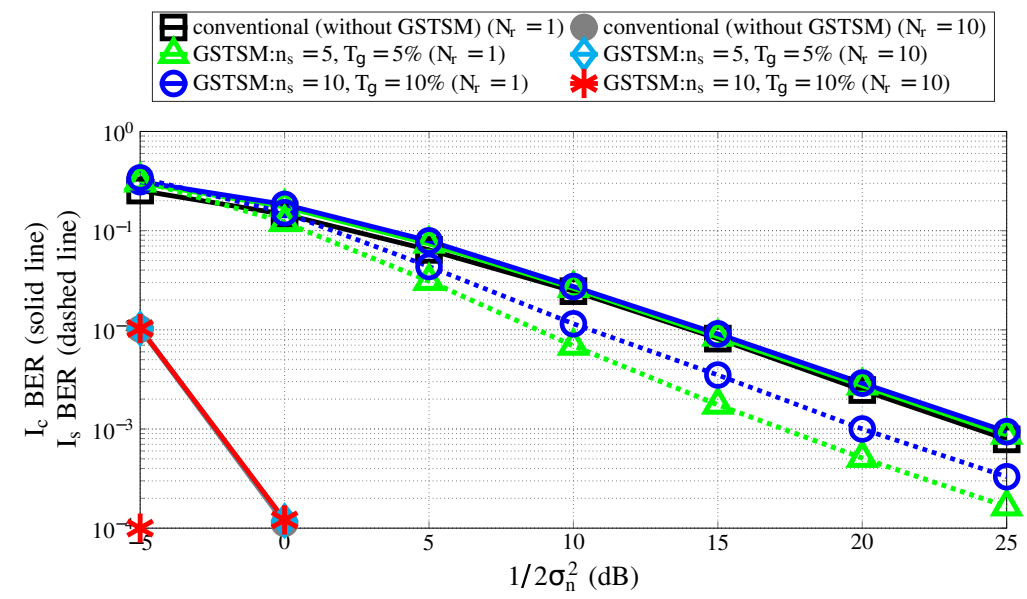

Fig. 3: The BER performance of the individual subchannels of GSTSM for several $n_{s}$ values (yielding a throughput gain of $T_{g}$ ) versus traditional scheme (without GSTSM) for $K=100$. For $I_{S}$ with $n_{s}=5$ and $N_{r}=10$, no error has been observed when transmitting $10^{5}$ information packets.

\section{Simulation Evaluations}

In this section, we present simulation results that illustrate the performance benefits of GSTSM. In Section V-A, we begin with the performance validation of the GSTSM's concept. The Bit-Error-Rate (BER) performance of GSTSM is shown for different scenarios and aforementioned pattern construction methods. Then, the performance gains of GSTSM against STSM [9][10] is presented in terms of achievable rates. Subsequently, in Section V-B, we show how GSTSM enables headerless and uncoordinated grant-free medium access for MTCs. We present the throughput gains of GSTSM-enabled rateless systems in collision-free and collision environments, and compare their performance with their header-based counterparts. For multi-user scenarios with collisions, the attainable throughput of GSTSM-enabled systems is further explored for joint multi-user detection and iterative detection/decoding receivers. The following assumptions have been made, unless stated otherwise. The MIMO channel is modelled as an uncorrelated frequency-flat Rayleigh channel, and the perfect knowledge of the channel is available at the receiver but not at the transmitter. In addition, it is assumed that each machine employs two transmitting antennae (with the transmitted power normalized to unity) and a single RF chain. Also, it is assumed that the conventional information is BPSK modulated, and the super-modulation employs patterns that are constructed based on Fixed Patterns. In Section V-B, where the GSTSM is used for rateless systems, it is assumed that Raptor rateless codes have been used to encode each packet. The Raptor codes are constructed based on an inner LT code according to Raptor RFC 5053 [31], and a rate 0.95 LDPC precode with left regular distribution (degree 3 nodes) and right Poisson. To recover the conventional information symbols, the LLRs of the received symbols are calculated from (11) and inserted into the softinformation-based Raptor decoder that executes sum-product algorithm [24] with forty iterations.

\section{A. Validation of the GSTSM Concept}

Fig. 3 shows the uncoded Bit-Error-Rate (BER) performance for the information subchannels $I_{c}$ and $I_{s}$, and for several sizes of super-modulated bit sequences $n_{s}$, resulting in a corresponding throughput increase of $T_{g}$. It is shown that the reliability of $I_{s}$ is significantly higher than that of $I_{c}$. It is also shown that the BER for $I_{s}$ is better for a smaller $n_{s}$, while the performance of $I_{c}$ remains unaffected even for a large value of $n_{s}$. Hence, a throughput gain of $10 \%$ can be obtained without affecting the BER of $I_{c}$. In addition, it can be observed that the reliability of both information subchannels is substantially enhanced for large-scale MIMO systems, e.g., using $N_{r}=10$, $T_{g}=10 \%$ can be attained even at very low SNRs. It can be observed that a highly-reliable information stream with a flexible-rate is provided by GSTSM. This cannot be achieved with traditional spatial modulation schemes. Moreover, Fig. 4 shows that the increase in $K$ significantly improves the BER of super-modulated bit stream due to the increase in the Euclidean distance between codewords. Still, even in the low block-length regime, the detection reliability of the conventionally modulated bit stream experiences negligible degradation that vanishes completely when exploiting the diversity gain of large-scale MIMO systems.

Fig. 5 illustrates a similar BER performance comparison for $n_{s}=5$, but in correlated channel conditions with a strong Rician $K_{R i c} .=3$ factor which is in the range of values measured in indoor communications [32]. Since in such correlated channel conditions there is no significant difference between the propagation characteristics of the transmitting antennae, GSTSM (similar to spatial modulation) cannot rely on the spatial dimension to detect the transmitted pattern. However, Fig. 5 shows that the super-modulated bits can still be detected with small performance degradation. This is achieved since GSTSM also exploits the temporal dimension of the transmitted signal. Again, the large antenna numbers at the receiver side results in a substantially improved reliability for both super-modulated and conventional information subchannels. In Fig. 6, the performance of GSTSM is evaluated in a single-input multiple-output system over an AWGN channel. It can be observed that even when the spatial dimension is not exploited, GSTSM still enables a reliable transmission of both information subchannels, just based on the introduced temporal correlation (i.e., phase rotations). The detection reliability 


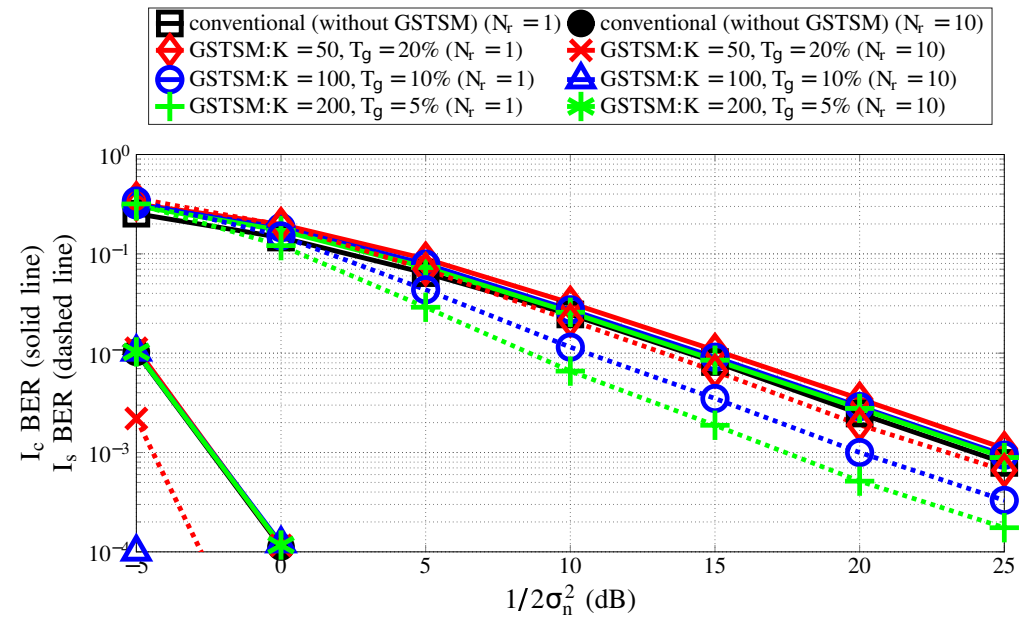

Fig. 4: The BER performance of the individual subchannels of GSTSM for $n_{s}=10$ and several $K$ values (yielding a throughput gain of $T_{g}$ ) versus traditional scheme (without GSTSM). For $I_{S}$ with $K=200$ and $N_{r}=10$, no error has been observed when transmitting $10^{5}$ information packets.

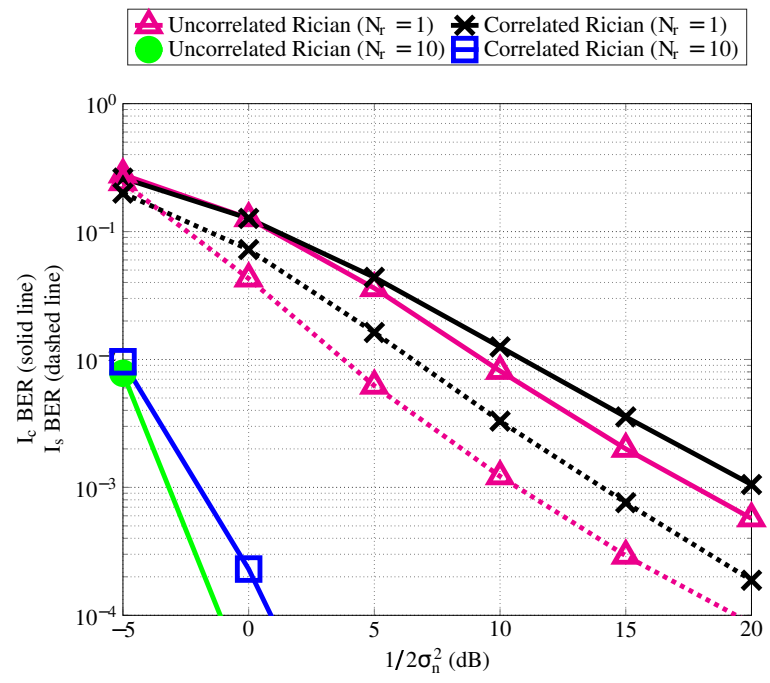

Fig. 5: Comparison of the BER performance of the individual subchannels of GSTSM for correlated and uncorrelated Rician fading channels with Rician $K_{\text {Ric. }}=3$ factor, and for $n_{s}=5$ and $K=100$. For $I_{s}$ with $N_{r}=10$, no error has been observed when transmitting $10^{4}$ information packets.

can again be substantially improved by exploiting receiver diversity.

Fig. 7 depicts the BER comparison of GSTSM schemes based on Fixed and PCA-based patterns for AWGN and fading channels. For $N_{r}=1$ over AWGN channel (Fig. 7(a)), it is shown that PCA-based GSTSM has superior performance, e.g., $2 d B$ gain at BER $\approx 10^{-4}$. For fading channels with $N_{r}=$ 1 (Fig. 7(c)), both Fixed and PCA schemes provide almost similar performance. Nevertheless, from Fig. 7(b) and 7(d), it can be observed that by exploiting receiver diversity in largescale MIMO systems, highly-reliable $I_{s}$ can be delivered for all cases, i.e., with $N_{r}=10$, no error is observed after transmitting $10^{4}$ data packets. Also, the associated $I_{c}$ stream of all schemes shown to yield similar performance as in traditional scheme (without GSTSM).

Moreover, Fig. 8 presents the achievable throughput of rateless systems based on GSTSM and STSM in collisionfree environment. A payload size of 1000 bits is used, and for

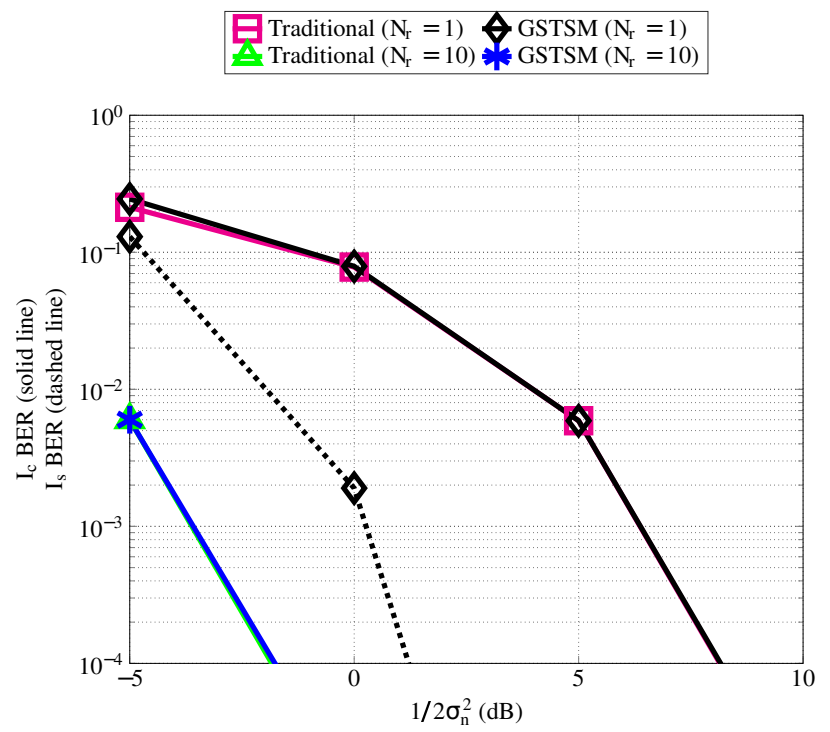

Fig. 6: Comparison of the BER performance of the individual subchannels of GSTSM with $n_{s}=5$ and $K=100$ for an AWGN channel. For $I_{s}$ with $N_{r}=$ 10 , no error has been observed after transmitting $10^{5}$ information packets.

transmission of each packet's SI, a $k_{s i}=9$-bit SI consisting of $k_{i}=6$ bits (to support 64 users as in LTE RACH) and $k_{o}=3$ bits is used. As can be seen, by utilizing advanced coding schemes, GSTSM obviates the need for exploiting temporal redundancy of space-time block codes as in the case of STSM, hence allows to substantially enhance the transmission rate that can reach over $85 \%$.

\section{B. Performance Evaluation of GSTSM for Grant-Free Com- munications}

As discussed in Section IV, GSTSM can be used in the context of grant-free communications to obviate the need for using packet headers for conveying packet's signature information. In this section, we elaborate on the application of GSTSM in this context. Fig. 9(a) compares the performance of the rateless systems where all SI bits are transmitted using GSTSM (i.e., headerless) versus the following four approaches 


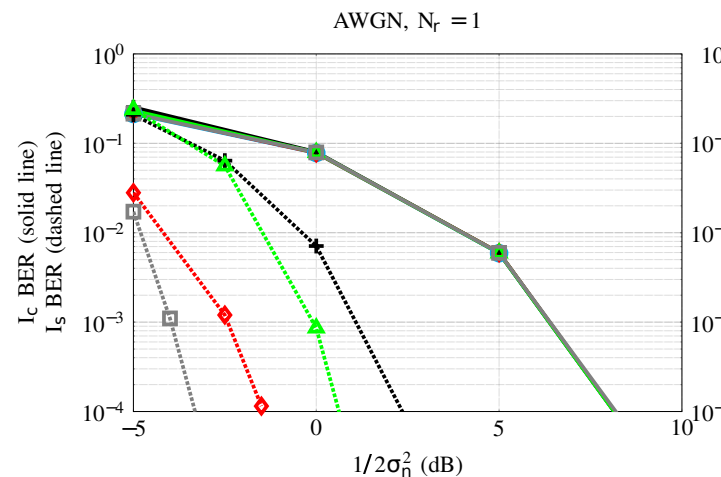

(a)

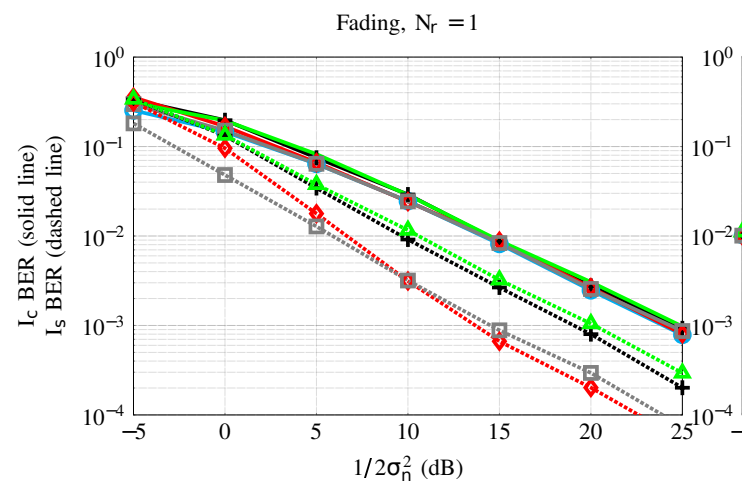

(c)

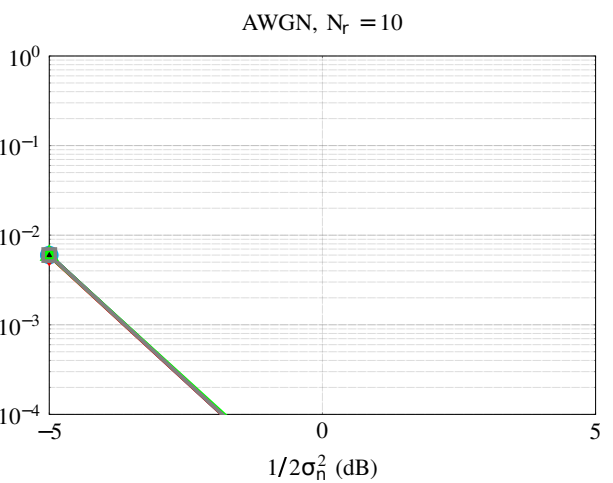

(b)

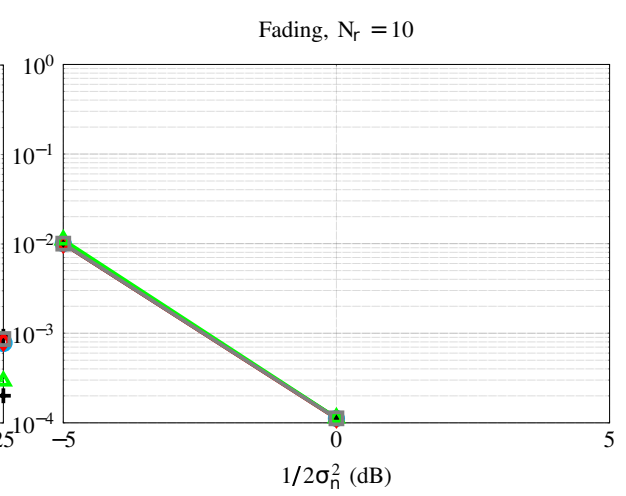

(d)

Fig. 7: Comparison of the BER performance of individual subchannels of GSTSM with $n_{s}=9$ for Fixed and PCA-based pattern construction methods, using $N_{r}=1$ and 10, and over: (a)-(b) AWGN channels, (c)-(d) fading channels. For all cases with $N_{r}=10$, no error has been observed for $I_{s}$ stream after transmitting $10^{4}$ information packets.

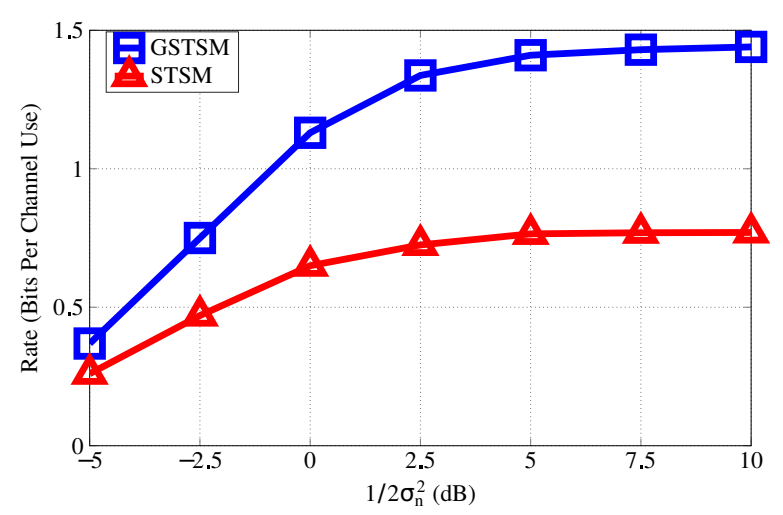

Fig. 8: Comparison of the achievable rate of rateless systems employing GSTSM and STSM schemes, for $K=200$ and $2 \times 2$ MIMO systems in collision-free environment.

for the transmission of $k_{s i}=9$-bit SI. The case where SI is perfectly known, and three header-based cases where SI bits are either mapped onto ZC sequences, or channel coded using a rate $\frac{9}{64}$ Polar Code (PC) or Convolutional Code (CC). Here, a payload size of 1000 bits is used, and a 9-bit SI consists of $k_{i}=6$ and $k_{o}=3$ bits.

To transmit $k_{s i}$ bits using $\mathrm{ZC}$ preambles, it is required to map them to $2^{k_{s i}}$ sequences with a minimum length of $2^{k_{s i}}$ samples. Therefore, preambles with a minimum of 512 samples are required for conveying $k_{s i}=9$-bit SI. As shown in Fig. 9(a), by using $N_{r}=1$, the GSTSM-enabled scheme approaches the ideal case and outperforms the header-based cases for a broad range of SNRs. In the case of large $N_{r}$, GSTSM yields similar performance to the ideal case, while also results superior performance compared to the headerbased cases across the whole $S N R$ range, enabling efficient headerless transmission. In addition, since in grant-free-based MTCs packet collisions are likely to happen [22], the appropriateness of GSTSM is also examined for the extreme case of uncoordinated transmissions. In this case, it is assumed that the transmitted packets of two machines are always colliding, similar to the two-user multiple access channel [33]. The attainable sum-rate for the various cases is shown in Fig. 9(b). In this case, it is assumed that the AP decodes each user's message independently by treating the interfering user's packet as noise. Hence, to detect each user's signal, the number of complex-valued multiplications for calculations of (10) and (11) remains the same as in the single-user scenario. It can be seen that for the case of $N_{r}=10$, the GSTSM-enabled scheme yields performance similar to the ideal case, while also results in up to $33 \%$ throughput gain compared with the best preamble-based method, i.e., the PC scheme.

In Fig. 10, the achievable rate of GSTSM scheme is shown for coordinated and uncoordinated transmissions of two users. For the former case, collision-free transmissions based on 


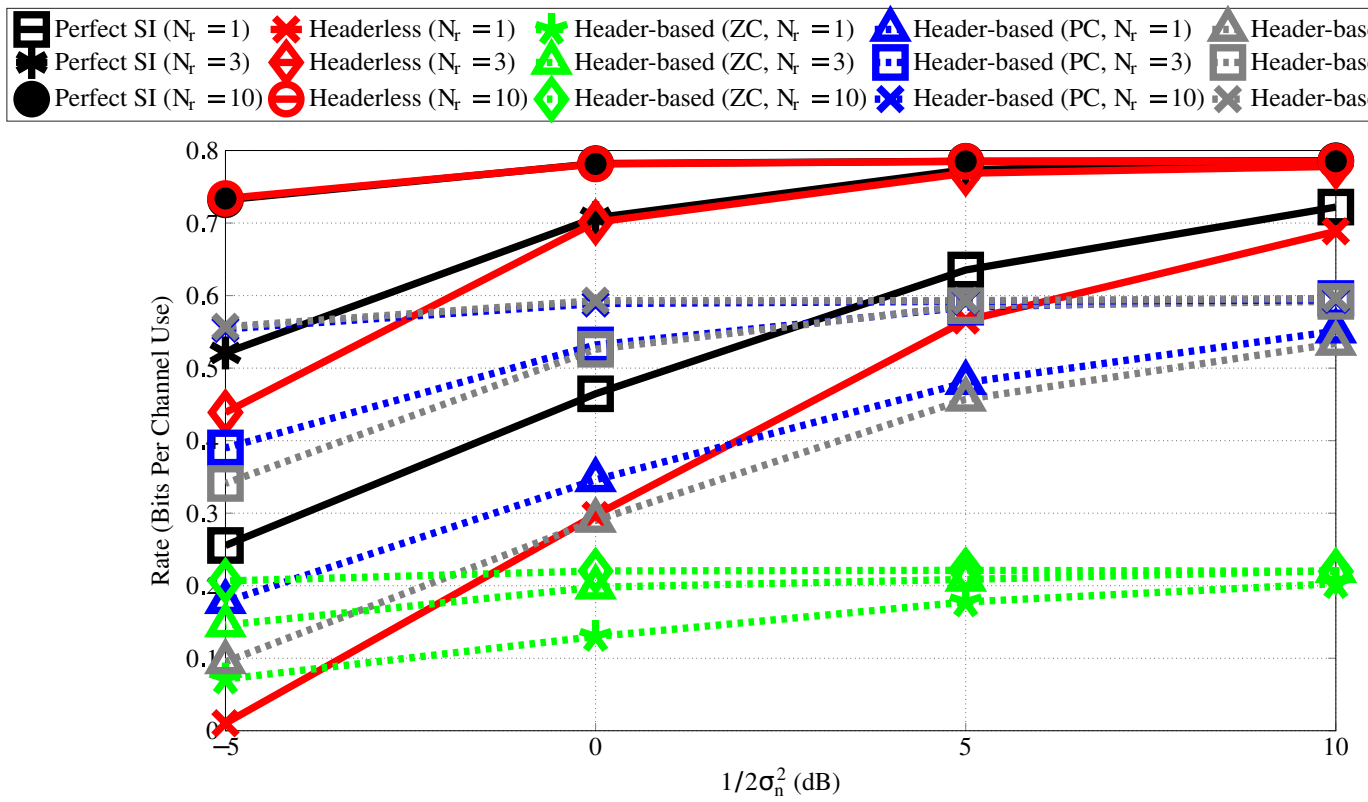

(a)

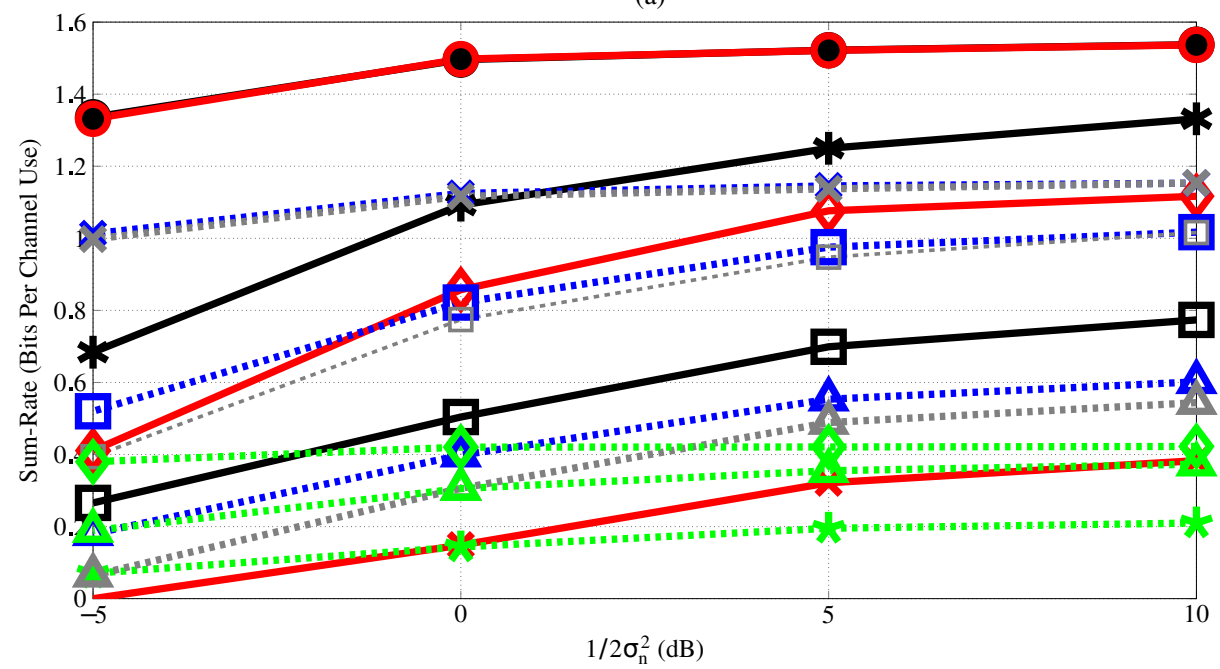

(b)

Fig. 9: Comparison of the achievable rate of the rateless systems employing headerless (GSTSM) scheme versus header-based schemes employing Zadoff-Chu (ZC), Polar-Coded(PC), and Convolutionally-Coded (CC) preambles, for $K=200$ and a varying number of receive antennae: (a) in multiuser collision-free environment, (b) multiuser environments with two colliding users.

TDMA is considered. For the later case, four schemes are considered, where joint and independent multi-user detection approaches are employed, with and without adopting iterative detection/decoding process. While in joint multi-user detection approach the ML problem as formulated in Section II is solved, for independent multi-user detection, it is solved for each user independently by taking into account the interfering machine's signal as noise. For both cases, the realization of iterative detection/decoding is in a way that when the information message of a user with better channel condition is successfully decoded, the related data packets are reconstructed at the AP, and their contribution from the received composite signal is cancelled. Similar to the concept of non-orthogonal multiple access [34], this allows the AP to re-try detection and decoding of the received signal that is not contaminated with inter-user interference. In the figure, the schemes with iterative and non- iterative process are denoted by "I" and "IN", respectively. Several observations can be made from the results. First, it can be seen that joint multi-user detection yields superior performance compared with the case in which users' signals are detected independently, e.g., without iterations and for $N_{r}=1$, the achievable gains can reach up to 2.5 times. However, by deploying a large $N_{r}$ at the AP, the performance penalty of the independent signal detection approach is significantly reduced, e.g., with $N_{r}=10$, its performance approaches that of the joint multi-user detection scheme. In addition, it can be seen that the iterative process yields throughput gains for both of the detection methods, e.g., for $N_{r}=1$, the throughput gain of up to $15 \%$ and $55 \%$ can be attained for joint and independent approaches, respectively. Still, for a large $N_{r}$, the performance of the non-iterative schemes gets closer to that of the iterative-based cases since the effect 

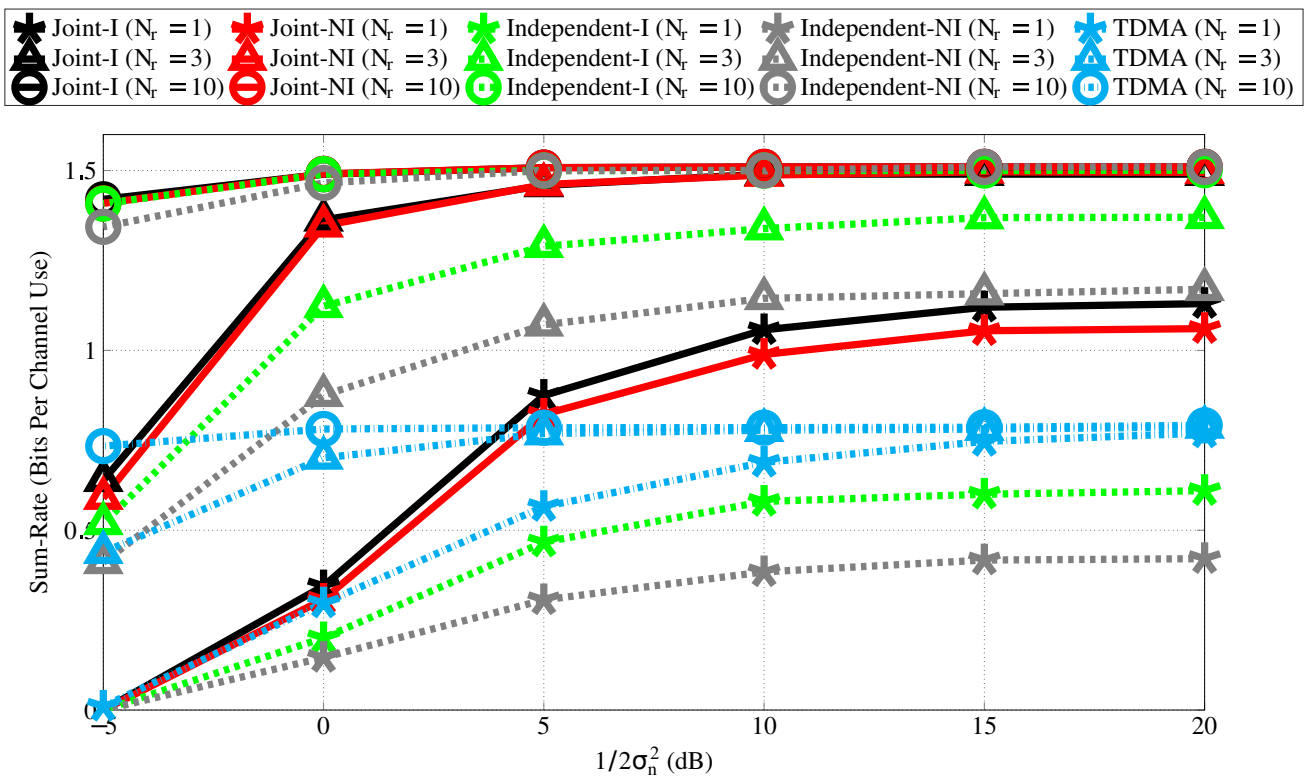

Fig. 10: Achievable sum-rate of the GSTSM-enabled rateless systems with two users based on uncoordinated access (using joint and independent multi-user detection, with and without iterative detection/decoding) versus TDMA. The schemes with iterative and non-iterative process are denoted by "I" and "IN", respectively. $K=200$, BPSK, and varying $N_{r}$ is assumed.

of inter-user interference diminishes by exploiting receiver diversity in large-scale MIMO systems. It is also shown that for $N_{r}=1$, uncoordinated transmissions based on joint and independent multi-user detection approaches yield superior and inferior performance compared with TDMA, respectively. However, the rateless properties of the transmitted information in uncoordinated transmissions allows to exploit the interfered received signals, and all four uncoordinated schemes result in superior performance compared to TDMA for $N_{r} \geq 3$, e.g., the achievable gains reach up to $90 \%$ for $N_{r}=10$.

Moreover, Fig. 11 (a) depicts the overall complexity requirements of the GSTSM's receiver with and without iterative detection/decoding approach, using joint and independent multi-user detectors. Considering the detection complexity, the number of incurred complex-valued multiplication operations for the joint detection approach is higher than the independent approach for each MIMO channel use, i.e., for two users with BPSK and super-modulation states as designed in Section III-A, the former and the later approaches require 64 and 20 complex-valued multiplication operations per MIMO channel use, respectively. On the other hand, the joint multi-user detector requires fewer data packets to be processed to reliably decode the transmitted message. Overall, it can be seen from the figure that the joint detection approaches require about $60 \%$ higher complexity compared with independent cases in the low $S N R$ regime (for both iterative and non-iterative schemes). In the high $S N R$ regime, the difference between the complexity of the two methods is not substantial. Moreover, it can be observed that for both joint and independent detection approaches, the difference between the complexity of the iterative and non-iterative approaches is not considerable. It is due to the fact that while iterative process adds computational complexity to the receiver, it allows to reduce the computations on the other hand since the decoding in such receivers converge faster than non-iterative receivers and therefore, it requires to process fewer packets. Furthermore, Fig. 11 (b) illustrates the processing latency of the GSTSM's receiver for the detection methods as in the previous figure. It can be seen that under good channel conditions, joint multi-user detection results in faster decoding. This gain comes at the cost of (negligible) computational complexity as shown in the previous figure. The iterative detection/decoding allows to process fewer packets for reliable decoding but this slightly increases the processing latency. However, by detecting the user's signals independently, the processing time taken by the iterative process is almost the same as the latency overhead for processing additional packets in non-iterative receivers. Hence, the overall processing latency for iterative and non-iterative receivers for the independent detection approach are almost similar.

In Fig. 12, the achievable sum-rate of a GSTSM-enabled scheme for two machines based on uncoordinated transmissions with the joint multi-user detection is presented for dense QAM constellations. Here, the super-modulation phase rotations are chosen from $\phi \in\left\{0, \frac{\pi}{14}\right\}$ (Section III). This figure shows that GSTSM can adopt dense constellations that offer prominent gains over a broad range of $S N R s$. This gain can reach up to 6 times compared with the BPSK when a massive number of receiving antennae is deployed at the AP.

\section{Conclusions}

We have introduced the concept of Generalized Space-Time Super-Modulation that enables the transmission of additional, flexible-rate, and highly-reliable information on top of the conventionally transmitted symbols. This is achieved without increasing the corresponding packet length, and without relying on temporal redundancy of space-time block codes. The additional information subchannel can efficiently operate 
目Joint-I目Joint-NI目Independent-I目Independent-NI

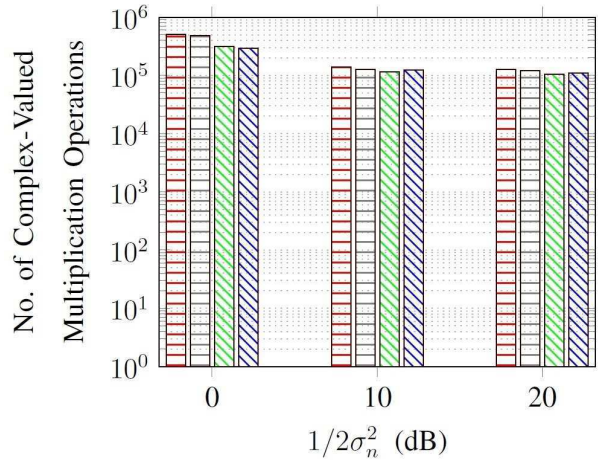

(a)

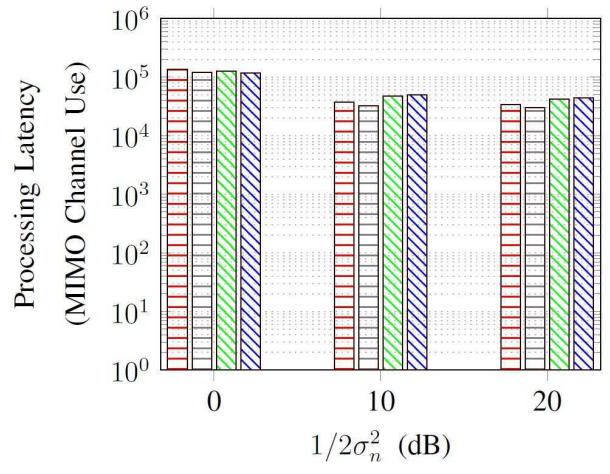

(b)

Fig. 11: GSTSM's receiver processing employing joint and independent multi-user detection schemes, with and without iterative detection/decoding approaches: (a) The overall complexity requirement, (b) The overall processing latency incurred. Rateless system with $K=200$ is used, and two colliding users with single receiver antenna is assumed.

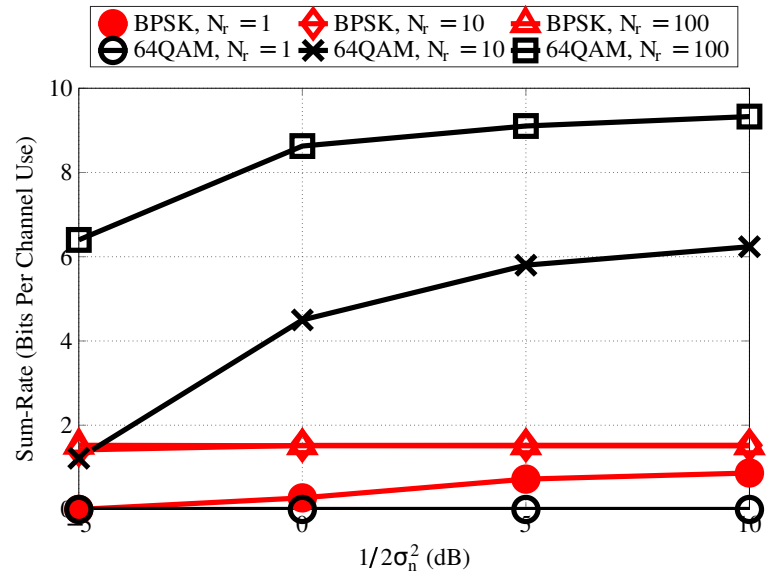

Fig. 12: Achievable sum-rate of the GSTSM-enabled rateless systems with two users and $K=200$, and for varying number of receiver antenna and dense QAM constellations.

even under highly correlated channel conditions or in AWGN channels. It is shown that GSTSM enables grant-free and headerless rateless transmissions for MTCs that may have several antennae at the machine side (even with one RF chain) and a large number of AP antennae. Compared with the schemes that require centralized coordination, it allows uncoordinated transmissions while also yielding significant throughput gains. Future work should investigate the potential applications of the GSTSM, e.g., the additional highly-reliable information stream of GSTSM can be exploited by applications that require bits with different reliability [35] such as video streaming applications.

\section{ACKNOWLEDGEMENT}

This research was been conveyed as part of the $\mathrm{PhD}$ studies of Dr Mehran. The principle ideas behind this research have been provided by Dr Nikitopoulos that has also been the principal supervisor of Dr Mehran. The authors would like to thank the members of the $5 \mathrm{G}$ and $6 \mathrm{G}$ Innovation Centre for their support.

\section{REFERENCES}

[1] F. Mehran and K. Nikitopoulos, "Generalized space-time supermodulation for headerless grant-free rateless multiple access," in Proc. IEEE GLOBECOM 2018.

[2] Y. Chen, et al., "Toward the standardization of non-orthogonal multiple access for next generation wireless networks," IEEE Commun. Mag., vol. 56, no. 3, pp. 19-27, Mar. 2018.

[3] J. Zhang, et al., "Cell-free massive MIMO: a new next-generation paradigm," IEEE Access, vol.7, pp.9978-9988, Aug.2019.

[4] Q. Wang, et al., "Non-orthogonal multiple access: a unfied perspective," IEEE Wireless Commun. Mag., vol. 25, no. 2, pp. 10-16, Apr. 2018.

[5] J. Zhang, et al., "PoC of SCMA-based uplink grant-free transmission in UCNC for 5G," IEEE J. Sel. Areas Commun., vol. 35, no. 6, pp. 13531362, Jun. 2017.

[6] C. Johnson, Long Term Evolution in Bullets, 2nd Edition, Jul. 2012.

[7] M. Jabi, et al., "AMC and HARQ: how to increase the throughput," IEEE Trans. Commun., vol. 66, no. 7, pp. 3136-3150, Jun. 2018.

[8] F. Mehran, et al., "Rateless wireless systems: gains, approaches, and challenges," in Proc. IEEE ChinaSIP 2015.

[9] K. Nikitopoulos, F. Mehran, and H. Jafarkhani, "Space-time supermodulation and its application to joint medium access and rateless transmission," in Proc. IEEE GLOBECOM 2016.

[10] K. Nikitopoulos, F. Mehran, and H. Jafarkhani, "Space-time supermodulation: concept, design rules, and its application to joint medium access and rateless transmission," IEEE Trans. Wireless Commun., vol.16, no.12, pp.8275-8288, Dec. 2017.

[11] V. Tarokh, H. Jafarkhani, and A. R. Calderbank, "Space-time block coding for wireless communications: performance results," IEEE J. Sel. Areas Commun., vol. 17, no. 3, pp. 451-460, Mar. 1999.

[12] _ _Space-time block codes from orthogonal designs," IEEE Trans. Inf. Theory, vol. 45, no. 5, pp. 1456-1467, Jul. 1999.

[13] S. M. Alamouti, "A simple transmit diversity technique for wireless communications," IEEE J. Sel. Areas Commun., vol. 16, no. 8, pp. 14511458, Oct. 1998

[14] E. Basar, et al., "Index modulation techniques for next-generation wireless networks," IEEE Access, vol. 5, pp. 16693-16746, Aug. 2017.

[15] R. Y. Mesleh, et al., "Spatial modulation," IEEE Trans. Veh. Technol, vol. 57, no. 4, pp. 2228-2241, Jul. 2008.

[16] G. Ungerboeck, "Trellis coded modulation with redundant signal sets, part I: introduction," IEEE Commun. Mag., vol. 25, no. 2, pp. 5-11, Feb. 1987.

[17] U. Wachsmann, et al., "Multilevel codes: theoretical concepts and practical design rules," IEEE Trans. Inf. Theory, vol. 45, no. 5, pp. 13611391, Jul. 1999.

[18] H. Jafarkhani and N. Seshadri, "Super-orthogonal space-time trellis codes," IEEE Trans. Inf. Theory, vol. 49, no. 4, pp. 937-950, Apr. 2003.

[19] M. Wen, et al., "Index modulation aided subcarrier mapping for dual-hop OFDM relaying," IEEE Trans. Commun., vol. 67, no. 9, pp. 6012-6024, Sep. 2019. 
[20] S. Sugiura, et al., "Generalized space-time shift keying designed for flexible diversity-, multiplexing- and complexity-tradeoffs," IEEE Trans. Wireless Commun., vol. 10, no. 4, pp. 1144-1153, Apr. 2011.

[21] M. A. Albreem, et al., "Massive MIMO detection techniques: a survey," IEEE Commun. Surveys Tuts., vol. 21, no. 4, pp. 3109-3132, Fourth Quarter 2019.

[22] C. Bockelmann, et al., "Massive machine-type communications in 5G: physical and MAC-layer solutions," IEEE Commun. Mag., vol. 54, no. 9, pp. 59-65, Sep. 2016.

[23] H. Jafarkhani, Space-Time Coding: Theory and Practice, Cambridge University Press, 2005.

[24] O. Etesami, et al., "Raptor codes on binary memoryless symmetric channels," IEEE Trans. Inf. Theory, vol. 52, no. 5, pp. 2033-2051, May 2006.

[25] K. Nikitopoulos, et al., "Approximate MIMO iterative processing with adjustable complexity requirements," IEEE Trans. Veh. Technol., vol. 61 , no. 2, pp. 639-650, Feb. 2012.

[26] R. G. Maunder, "The implementation challenges of Polar codes", AccelerComm, Feb. 2018.

[27] K. Nikitopoulos, et al., "Geosphere: consistently turning MIMO capacity into throughput," in Proc. ACM SIGCOMM 2014.

[28] K. Nikitopoulos, et al., "Massively parallel tree search for highdimensional sphere decoders," IEEE Trans. Parallel Distrib. Syst., vol. 30, no. 10, pp. 2309-2325, Oct. 2018.

[29] J. M. V.-Gutierrez, et al., "Sequence sets in wireless communication systems: a survey," IEEE Commun. Surveys Tuts., vol. 19, no. 2, pp. 1225-1248, Second Quarter 2017.

[30] N. Jiang, et al., "Analyzing random access collisions in massive IoT networks," IEEE Trans. Wireless Commun., vol. 17, no. 10, pp. 68536870, Oct. 2018.

[31] M. Luby, et al., "Raptor forward error correction scheme for object delivery," RFC 5053, Oct. 2007.

[32] R. Mesleh, et al., "Trellis coded spatial modulation," IEEE Trans. Wireless Commun., vol.9, no.7, pp.2349-2361, Jul.2010.

[33] D. Tse and P. Viswanath, Fundamentals of Wireless Communication, Cambridge, UK: Cambridge University Press, 2005.

[34] X. Zou, et al.,"Trellis-coded non-orthogonal multiple access," IEEE Wireless Commun. Lett., vol.9, pp.538-542, Apr. 2020.

[35] B. Masnick, et al., "On linear unequal error protection codes," IEEE Trans. Inf. Theory, vol. IT-3, pp. 600-607, Oct. 1967.

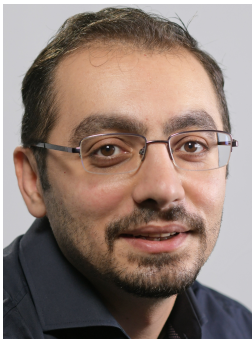

Farhad Mehran received the M.Eng. degree in Electronic and Communications Engineering from the University of Birmingham, U.K., in 2009, and Ph.D. degree from the University of Surrey, Guildford, U.K., in 2020. In 2016, he joined the Wireless Research team at BT Research and Innovation, Adastral Park, U.K., where he is working on physical and systems aspects of wireless communications. His research interests include channel coding, MIMO systems, and multiple access techniques.

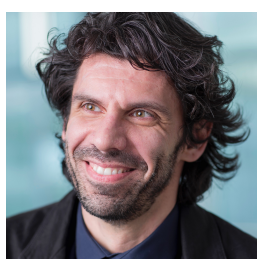

Konstantinos Nikitopoulos is a Senior IEEE Member and a Reader in signal processing for communication systems with the Electrical and Electronic Engineering Department, Institute for Communication Systems, University of Surrey, Guildford, U.K. $\mathrm{He}$ is a member of the $5 \mathrm{G}$ and $6 \mathrm{G}$ Innovation Centre, where he leads the "Theory and Practice of Advanced Concepts in Wireless Communications" work area, and is the Director of the Wireless Systems Laboratory of the Institute for Communication Systems. He has held research positions with RWTH Aachen University, Germany, the University of California at Irvine, USA, and University College London, U.K. He was a recipient of the prestigious First Grant of the U.K.'s Engineering and Physical Sciences Research Council and the Principal Investigator of several research projects, including the AutoAir I and II projects, of the U.K.'s 5G Testbeds and Trials Programme.

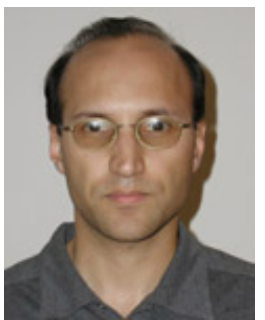

Hamid Jafarkhani is a Chancellor's Professor at the Department of Electrical Engineering and Computer Science, University of California, Irvine, where he is also the Director of Center for Pervasive Communications and Computing, the Co-Director of Networked Systems Program, and the ConexantBroadcom Endowed Chair. He is the 2020-2022 elected Faculty Chair of the UCI School of Engineering. He was a Visiting Scholar at Harvard University in 2015 and a Visiting Professor at California Institute of Technology in 2018. Among his awards are the NSF Career Award, the UCI Distinguished Mid-Career Faculty Award for Research, the School of Engineering Excellence in Research Senior Career Award, the IEEE Marconi Prize Paper Award in Wireless Communications, the IEEE Communications Society Award for Advances in Communication, the IEEE Wireless Communications Technical Committee Recognition Award, and the IEEE Eric E. Sumner Award.

Dr. Jafarkhani is listed as an ISI highly cited researcher. According to the Thomson Scientific, he is one of the top 10 most-cited researchers in the field of "computer science" during 1997-2007. He is the 2017 Innovation Hall of Fame Inductee at the University of Maryland's School of Engineering. He is a Fellow of AAAS, an IEEE Fellow, and the author of the book "Space-Time Coding: Theory and Practice." 